\title{
TOPOLOGY IN NONLINEAR EXTENSIONS OF HYPERNUMBERS
}

\author{
M. S. BURGIN \\ Received 9 September 2004
}

Modern theory of dynamical systems is mostly based on nonlinear differential equations and operations. At the same time, the theory of hypernumbers and extrafunctions, a novel approach in functional analysis, has been limited to linear systems. In this paper, nonlinear structures are introduced in spaces of real and complex hypernumbers by extending the concept of a hypernumber. In such a way, linear algebras of extended hypernumbers are built. A special topology of conical neighborhoods in these algebras is introduced and studied. It is proved that the space of all extended real hypernumbers is Hausdorff. This provides uniqueness for limits what is very important for analysis of dynamical systems. It is also proved that construction of extended real hypernumbers is defined by a definite invariance principle: the space of all extended real hypernumbers is the biggest Hausdorff factorization of the sequential extension of the space of all real numbers with the topology of conical neighborhoods. In addition, this topology turns the set of all bounded extended real hypernumbers into a topological algebra. Other topologies in spaces of extended hypernumbers are considered.

\section{Introduction}

Discrete dynamics reflects a new emerging tendency towards utilization of iterative mathematical models to describe the behavior of complex systems. The theory of hypernumbers and extrafunctions (cf., e.g., Burgin [7]) provides tools and structures for discrete dynamics. The first advantage of this theory is a possibility to construct new dynamical models. If a system $R$ is represented by means of its state space which is usually a Euclidean, Hilbert, or Banach space, then trajectories in this space reflect dynamics of $R$. It is natural to represent such trajectories by systems (vectors, matrices, $n$-dimensional matrices, etc.) of hypernumbers. This is especially convenient when a researcher considers discrete time or the trajectory of a system is given (by measurement or computation) at discrete moments of time. Hypernumbers that represent trajectories show asymptotic behavior of the system.

In general, the theory of hypernumbers and extrafunctions emanated from physically directed thinking and was derived by a natural extension of the classical approach to the real number universe construction. Namely, an important class of problems that appear 
in contemporary physics and involve infinite values inspired this theory. As it is known, many mathematical models, which are used in modern theories of elementary particles (such as gauge theories), imply divergence of analytically calculated properties of physical systems. The simplest example is the case of a free electron when its interaction with photons changes the energy of the electron so that the energy becomes infinite (in a model). Mathematical investigation of various physical problems gives rise to divergent integrals and series that are such mathematical constructions that have, in some sense, infinite values. However, physical measurements give, as the result, only finite values. That is why many methods of divergence elimination (regularization), that is, of elimination of infinity, have been elaborated. Nevertheless, the majority of them were not well grounded mathematically because they utilized operations with formal expressions that had neither mathematical nor physical meaning. Moreover, there are such models in physics that contain infinities that cannot be eliminated by these methods based on existing mathematical theories. Only in the theory of hyperintegration, based on the theory of hypernumbers, all divergent integrals and series that appear in the calculations with physical quantities become correctly grounded as strict mathematical objects.

The second advantage of the theory of hypernumbers and extrafunctions is a possibility to build a new calculus for discrete processes. As Gontar [17] writes, “... the calculus of infinitesimals - the theory of differential equations, to date the only mathematical apparatus for describing dynamics - will seemingly have to be modified before it can be used to describe the nonlinear dynamics of systems with chaotic behavior. The problem of the existence of a $\lim \Delta x / \Delta t$ for $\Delta t \rightarrow 0$ is particularly crucial in the study of chaos. Is the language of differential calculus which appears in the mathematical formulation of all dynamical laws truly so universal? Or is it possible to construct a mathematical tool or even a new calculus for describing dynamics without using derivatives and free of the contradiction between the continuous time and space of differential equations and the discrete process of calculation?"

In the context of hypernumbers, it is not necessary to take the limit for finding the derivative. Instead of arbitrary converging sequences, we can use hypernumbers and approximations of points and functions for the same goal. Namely (cf. Burgin $[4,7]$ ), given a point $a$ and a real or complex function $f(x)$, we take an approximation $I=$ $\left\{\left(a_{m}, b_{m}\right) ; n \in \omega\right\}$ of $a$ and approximation $F=\left\{f_{n} ; n \in \omega\right\}$ of $f(x)$ and define the partial extraderivative $\partial / \partial F, I f_{x}$ of $f$ at $x$ with respect to $F$ and $I$ equal to the hypernumber $\operatorname{Hn}\left(\Delta_{m} f_{n} / \Delta_{m} x\right)_{(m, n) \in \omega} 2$, where $\Delta_{m} x=b_{m}-a_{m}$ and $\Delta_{m} f_{n}=f_{n}\left(b_{m}\right)-f_{n}\left(a_{m}\right)$. When it is possible to obtain (to measure or compute) values of the initial function $f(x)$ and the value $x$, we do not need approximations, and the extraderivative of $f$ at $x$ has the simpler form ${ }^{\partial} / \partial I f_{x}=\operatorname{Hn}\left(\Delta_{m} f / \Delta_{m} x\right)_{m \in \omega}$. This approach is an extension of the classical differentiation because in the case of continuous spaces and existing limits, we get exactly the classical derivative.

At the same time, when we take a general case of approximations, the differential calculus of extraderivatives is relevant to discrete processes and includes as particular cases, or as subcalculi, different calculi of finite differences (Boole [3]; Milne-Thomson [34]; Richardson [39]; Jordan [21]; Spiegel [43]), as well as the quantum calculus developed by Kac and Cheung [22]. 
The third advantage of the theory of hypernumbers and extrafunctions is a possibility to solve much more differential equations than it is possible to solve with classical methods and even with distributions (Burgin and Ralston [11]). However, the main advance took place only with linear differential equations due to the fact that there were essential problems with multiplication of hypernumbers and extrafunctions. Spaces of hypernumbers and extrafunctions form only linear spaces over the field of real/complex numbers. There is no general multiplication in these sets. In such spaces, it is possible to multiply only very limited sets of hypernumbers and extrafunctions. The situation appeared to be very similar to the problem of distribution multiplication due to the fact that the theory of extrafunctions encompasses distribution theory.

At the same time, many problems in physics and PDE demand multiplication (cf., e.g., Oberguggenberger [37] or Nicolis and Prigogine [36]). Only structures with multiplication are relevant for generating and describing complex nonlinear phenomena, including chaotic regimes and fractals. This caused different mathematicians to introduce various constructions for multiplication of distributions. As Oberguggenberger [37] writes, "first attempts in defining nonlinear operations within distribution theory go back to the early fifties, pressed by the renormalization problem in quantum field theory as it was seen then." The most developed constructions of differential algebras that contained distributions were presented in the works by Berg [1], Burgin [6], Colombeau [13], Delcroix and Scarpalezos [14], Egorov [15], Fisher [16], B. H. Li and Y. Q. Li [29], Oberguggenberger [37], Rosinger [40,41], and others, and a new theory of generalized functions has been developed.

In this work, we define multiplication in the setting of hypernumbers and extrafunctions (Section 2) and study topological properties of this system (Section 3). To be able to use operation of multiplication, we extend the system of hypernumbers $\mathbb{R}_{\omega}$ studied in Burgin $[4,7,8,9]$, Burgin and Ralston [11] to the system of E-hypernumbers $\mathbf{E R}_{\omega}$. This extension gives means for multiplication in vast classes of E-hypernumbers, preserving at the same time good topological properties. In turn, this provides a possibility to study nonlinear transformations and nonlinear dynamical systems for hypernumbers.

An important property of mathematical spaces used for modeling physical systems is their topology. As history of physics shows, topology of underlying spaces is inherently connected with properties of physical systems (cf., e.g., Nash [35], Witten [44, 45]). An important field of modern quantum physics is formed by topological quantum field theories (see [23]). Inappropriate topology in the state space can result in insolvability of such simple partial differential equations as $\partial / \partial t f=c$, where $c$ is a constant (Oberguggenberger [37]).

In addition, the topological structure of hypernumbers and extrafunctions has an impact on differential calculus in the hypernumber universe: essential properties of differentiation rest on this topology in a similar way as many features of the classical calculus are dependent on the topology of the real line. An example of such a feature is uniqueness of the limit of a sequence.

That is why Section 3 of the paper deals with topology in the space of E-hypernumbers. The goal is to have a "good" topology such as, for example, the Hausdorff topology. If we take for E-hypernumbers topology determined by spherical neighborhoods, which gave 
Hausdorff topology for spaces of hypernumbers, we easily find that for E-hypernumbers the corresponding topology is not Hausdorff. Thus, we introduce another topology for E-hypernumbers that is defined by conical neighborhoods.

It is proved (Theorem 3.24) that the space of all E-hypernumbers is Hausdorff in this topology. This provides uniqueness for limits what is very important for analysis. In addition to this, it is proved (Theorem 3.25) that construction of E-hypernumbers is defined by a definite invariance principle: the space of all real E-hypernumbers is the biggest Hausdorff factorization of the sequential extension of the space of all real numbers with the topology defined by conical neighborhoods.

Thus, we achieve the goal to build a sufficiently general hypernumber algebra, preserving at the same time good topological properties.

\section{Denotations.}

(1) $\mathbb{N}$ is the set of all natural numbers.

(2) $\omega$ is the sequence of all natural numbers.

(3) $\mathbb{R}$ is the set of all real numbers.

(5) If $a$ is a real number, then $|a|$ is its absolute value.

(6) $\mathbb{R}^{+}$is the set of all nonnegative real numbers.

(7) $\mathbb{R}^{++}$is the set of all positive real numbers.

(8) $\mathbb{R}^{\omega}$ is the set of all sequences of real numbers.

(9) $\mathbf{i}^{\mathbf{n}}=\left(1 / i^{n}\right)_{i \in \omega} ; \mathbf{0}=\left(a_{i}=0\right)_{i \in \omega} ; \mathbf{e}_{k}=\left(e^{-k i}\right)_{i \in \omega}$.

(10) If $a=\left(a_{i}\right)_{i \in \omega}$, then $|a|=\left(\left|a_{i}\right|\right)_{i \in \omega}$.

(11) $\mathbb{C}$ is the set of all complex numbers.

(12) $\mathbb{C}^{\omega}$ is the set of all sequences of complex numbers.

(13) If $\mathbf{F}$ is a set of complex functions, then $\mathbf{F} \mathbb{C}^{\omega}$ is the set of all $\mathbf{F}$-moderate sequences of complex numbers.

(14) $\mathbf{D} \mathbb{R}^{\omega}$ is the set of all $\mathbf{D}$-moderate sequences of real numbers, where $\mathbf{D}=\left\{k x^{n} ; k \in\right.$ $\left.\mathbb{R}^{++}, n \in \mathbb{N}\right\}$.

(15) $\mathbf{E}^{-1} \mathbb{R}^{\omega}$ is the set of all $\mathbf{E}^{-1}$-moderate sequences of real numbers, where $\mathbf{E}^{-1}=$ $\left\{e^{-k x} ; k \in \mathbb{R}^{++}\right\}$.

(16) $\mathbb{R}_{\omega}$ is the set of all real hypernumbers.

(17) $\mathbf{E R}_{\omega}$ is the set of all real E-hypernumbers.

(18) $\mathbf{E}^{\mathrm{D}} \mathbb{R}_{\omega}$ is the set of all D-moderate real E-hypernumbers.

(19) $\mathbb{C}_{\omega}$ is the set of all complex hypernumbers.

(20) $\mathbf{E} \mathbb{C}_{\omega}$ is the set of all complex $\mathbf{E}$-hypernumbers.

(21) $\mathbf{E}^{\mathbf{D}} \mathbb{C}_{\omega}$ is the set of all $\mathbf{D}$-moderate complex $\mathbf{E}$-hypernumbers.

\section{Moderate sequences, majorants, and extended hypernumbers}

We consider the set $\mathbb{R}^{\omega}=\left\{\left(a_{i}\right)_{i \in \omega} ; a_{i} \in \mathbb{R}\right\}$ of all sequences of real numbers and define hypernumbers studied in Burgin $[4,7,8]$.

Definition 2.1. For arbitrary sequences $a=\left(a_{i}\right)_{i \in \omega}, b=\left(b_{i}\right)_{i \in \omega} \in \mathbb{R}^{\omega}$,

$$
a \sim b \longleftrightarrow \lim _{i \rightarrow \infty}\left|a_{i}-b_{i}\right|=0 .
$$

The relation $\sim$ is an equivalence. This allows us to define real hypernumbers. 
Definition 2.2. Classes of the equivalence $\sim$ are called real hypernumbers and their set is denoted by $\mathbb{R}_{\omega}$.

Any sequence $a=\left(a_{i}\right)_{i \in \omega}$ determines a hypernumber $\alpha=\operatorname{Hn}\left(a_{i}\right)_{i \in \omega}$. Real hypernumbers are sets of equivalent sequences of real numbers like rational numbers are sets of equivalent fractions or real number sets of equivalent fundamental sequences of rational numbers. In a similar way, real hypernumbers are defined as sets of equivalent sequences of real numbers.

However, there is no natural multiplication in the set $\mathbb{R}_{\omega}$. To overcome this shortcoming, we extend the space of hypernumbers.

Definition 2.3. An (increasing) function $f: \mathbb{R}^{++} \rightarrow \mathbb{R}^{++}$is called an (increasing) majorant [k-majorant] of a sequence $\left(a_{i}\right)_{i \in \omega}$ if $f(i)>\left|a_{i}\right|$ [if $\left.f(i)>\left|a_{i}\right|+k\right]$ for almost all $i \in \mathbb{N}$, meaning "all but finitely many $i$ from $\mathbb{N}$."

Definition 2.4. A $k$-majorant of a sequence $\left(a_{i}\right)_{i \in \omega}$ with an arbitrary $k$ is also called its strict majorant.

LEMMA 2.5. Any strict majorant of a sequence is a majorant of the same sequence. Let $f(i)$ be an increasing function.

Definition 2.6. A sequence $\left(a_{i}\right)_{i \in \omega}$ is called (strictly) $f$-moderate if $f(i)$ is its increasing (strict) majorant.

LEMMA 2.7. If $f(i)$ is a (strict) majorant of a sequence $\left(a_{i}\right)_{i \in \omega}$ and $g(i) \geq f(i)$ for almost all $i \in \mathbb{N}$, then $g(i)$ is a (strict) majorant of the sequence $\left(a_{i}\right)_{i \in \omega}$.

LEMMA 2.8. The concept of an (increasing) strict majorant is invariant with respect to the choice of a sequence that represents a hypernumber, that is, if $f(i)$ is an (increasing) strict majorant of a sequence $\left(a_{i}\right)_{i \in \omega}$ and $\operatorname{Hn}\left(a_{i}\right)_{i \in \omega}=\operatorname{Hn}\left(b_{i}\right)_{i \in \omega}$, then $f(i)$ is an (increasing) strict majorant of a sequence $\left(b_{i}\right)_{i \in \omega}$.

Proof. Properties of real numbers imply the following sequence of equalities and inequalities: $b_{i}=b_{i}-a_{i}+a_{i}=\left(b_{i}-a_{i}\right)+a_{i} \leq\left|b_{i}-a_{i}\right|+a_{i}$. By the definition of a strict majorant, there are $k \in \mathbb{R}^{++}$and $m \in \omega$ such that for all $i>m$, we have $\left|a_{i}-b_{i}\right|<f(i)+k$ and by the definition of a hypernumber, there is $n \in \omega$ such that for all $i>n$, we have $\left|b_{i}-a_{i}\right|<k$. Consequently, for all $i>\max (m, n)$, we have $b_{i} \leq\left|b_{i}-a_{i}\right|+a_{i} \leq f(i)+2 k$. It means that $f(i)$ is an (increasing) strict majorant of a sequence $\left(b_{i}\right)_{i \in \omega}$.

The lemma is proved.

Remark 2.9. The concept of a majorant is not invariant with respect to the choice of a sequence that represents a hypernumber, that is, if a function $f(i)$ is a majorant of a sequence $\left(a_{i}\right)_{i \in \omega}$ and $\operatorname{Hn}\left(a_{i}\right)_{i \in \omega}=\operatorname{Hn}\left(b_{i}\right)_{i \in \omega}$, then $f(i)$ is not necessarily majorant of a sequence $\left(b_{i}\right)_{i \in \omega}$, as the following example demonstrates.

Example 2.10 (J. Ralston). By the definition, $1=\operatorname{Hn}(1+1 / i)_{i \in \omega}=\operatorname{Hn}(1-1 / i)_{i \in \omega}$. Then the function $f(i) \equiv 1$ is a majorant of a sequence $(1-1 / i)_{i \in \omega}$ but is not a majorant of a sequence $(1+1 / i)_{i \in \omega}$.

The result of Lemma 2.7 allows us to introduce increasing majorants for hypernumbers. 
Definition 2.11. An (increasing) function $f(i)$ is called an (increasing) majorant of a hypernumber $\alpha=\operatorname{Hn}\left(a_{i}\right)_{i \in \omega}$ if $f(i)$ is an (increasing) strict majorant of the sequence $\left(a_{i}\right)_{i \in \omega}$.

Definition 2.12. A hypernumber $\alpha=\operatorname{Hn}\left(a_{i}\right)_{i \in \omega}$ is called $f$-moderate if $f(i)$ is its majorant.

The set of all $f$-moderate sequences of real numbers is denoted by $f \mathbb{R}^{\omega}$, the set of all strictly $f$-moderate sequences of real numbers is denoted by $s f \mathbb{R}^{\omega}$, and the set of all $f$-moderate real hypernumbers is denoted by $f \mathbb{R}_{\omega}$.

Lemma 2.13. A hypernumber $\alpha$ is $f$-moderate if and only if $f(i)$ is a strict majorant of any sequence $\left(a_{i}\right)_{i \in \omega}$ such that $\alpha=\operatorname{Hn}\left(a_{i}\right)_{i \in \omega}$.

CoRollary 2.14. If $f(i)$ is a majorant of a hypernumber $\alpha$ and $g(i) \geq f(i)$ for almost all $i \in \mathbb{N}$, then $g(i)$ is a majorant of the hypernumber $\alpha$.

Corollary 2.15. If $g(i) \geq f(i)$ for almost all $i \in \mathbb{N}$, then $f \mathbb{R}^{\omega} \subseteq g \mathbb{R}^{\omega}, s f \mathbb{R}^{\omega} \subseteq \operatorname{sg} \mathbb{R}^{\omega}$, and $f \mathbb{R}_{\omega} \subseteq g \mathbb{R}_{\omega}$.

Let $\mathbf{F}=\left\{f_{t}(i) ; t \in T\right\}$ be a class of functions from $\mathbb{R}^{++}$into $\mathbb{R}^{++}$.

Definition 2.16. A sequence $\left(a_{i}\right)_{i \in \omega}$ is called (strictly) F-moderate if some function $f(i)$ from $\mathbf{F}$ is its (strict) majorant.

For the class F, the set of all F-moderate sequences is denoted by $\mathbf{F} \mathbb{C}^{\omega}$ and the set of all strictly $\mathbf{F}$-moderate sequences is denoted by $s \mathbf{F} \mathbb{C}^{\omega}$.

Let $\mathbf{G}$ be also a class of functions from $\mathbb{R}^{++}$into $\mathbb{R}^{++}$.

Proposition 2.17. If for any function $f$ from $\mathbf{F}$ there is a function $h$ from $\mathbf{G}$ such that $h(i) \geq f(i)$ for almost all $i \in \mathbb{N}$, then any (strictly) F-moderate sequence $\left(a_{i}\right)_{i \in \omega}$ is (strictly) G-moderate.

It is possible that for different classes $\mathbf{F}$ and $\mathbf{G}$ of increasing functions, classes $\mathbf{F} \mathbb{R}^{\omega}$ and $\mathbf{G} \mathbb{R}^{\omega}$ coincide, as well as classes $\mathbf{F} \mathbb{R}_{\omega}$ and $\mathbf{G R}_{\omega}$.

Definition 2.18. A hypernumber $\alpha=\operatorname{Hn}\left(a_{i}\right)_{i \in \omega}$ is called F-moderate if some function $f(i)$ from $\mathbf{F}$ is its majorant.

Example 2.19. Given an increasing function $f(x)$ from $\mathbb{R}^{++}$into $\mathbb{R}^{++}$with $f(2)>1$, we can consider the set $\mathbf{D}(f)=\left\{k f^{n} ; k, n \in \mathbb{N}\right\}$ as the class $\mathbf{F}$. In particular, we have the set $\mathbf{D}=\left\{k x^{n} ; k, n \in \mathbb{N}\right\}$ of powers of the variable $x$.

Example 2.20. Given an increasing function $f(x)$ from $\mathbb{R}^{++}$into $\mathbb{R}^{++}$, we can consider the set $\mathbf{P}_{\mathbf{w}}(f)$ of all polynomials of $f$ with whole number coefficients as the class $\mathbf{F}$. In particular, we have the set $\mathbf{P}_{\mathbf{w}}$ of all polynomials of $f$ with whole number coefficients.

Lemma 2.21. A sequence $\left(a_{i}\right)_{i \in \omega}$ is strictly $\mathbf{D}(f)$-moderate (strictly $\mathbf{D}$-moderate) if and only if it is $\mathbf{D}(f)$-moderate ( $\mathbf{D}$-moderate).

Proof. By the definition, for any set of functions F, if a sequence $\left(a_{i}\right)_{i \in \omega}$ is strictly Fmoderate, then this sequence is F-moderate. Let $\left(a_{i}\right)_{i \in \omega}$ be a $\mathbf{D}(f)$-moderate sequence. Then there are numbers $k \in \mathbb{R}^{++}$and $n, m \in \omega$ such that for all $i>m$, we have $\left|a_{i}\right|<$ $k f(i)^{n}$. As $f(x)$ is an increasing function and $f(2)>1$, there is a number $h \in \mathbb{R}^{++}$such 
that for all $i>m \geq 2$, we have $h f(i)^{n}>1$. Then taking $q=k+h$, we have $q f(i)^{n}=k f(i)^{n}+$ $h f(i)^{n}>\left|a_{i}\right|+1$ for all $i>m$. It means that $\left(a_{i}\right)_{i \in \omega}$ is a strictly $\mathbf{D}(f)$-moderate sequence.

Proposition 2.17 and Lemma 2.21 imply the following result.

Lemma 2.22. A real hypernumber $\alpha$ (a sequence $\left.\left(a_{i}\right)_{i \in \omega}\right)$ is $\mathbf{D}(f)$-moderate (D-moderate) if and only if it is $\mathbf{P}_{\mathbf{w}}(f)$-moderate ( $\mathbf{P}_{\mathbf{w}}$-moderate), that is, $\mathbf{D}(f) \mathbb{R}^{\omega}=\mathbf{P}_{\mathbf{w}}(f) \mathbb{R}^{\omega}, \mathbf{D} \mathbb{R}^{\omega}=$ $\mathbf{P}_{\mathbf{w}} \mathbb{R}^{\omega}, \mathbf{D}(f) \mathbb{R}_{\omega}=\mathbf{P}_{\mathbf{w}}(f) \mathbb{R}_{\omega}$, and $\mathbf{D} \mathbb{R}_{\omega}=\mathbf{P}_{\mathbf{w}} \mathbb{R}_{\omega}$.

Corollary 2.23. A sequence $\left(a_{i}\right)_{i \in \omega}$ is strictly $\mathbf{P}_{\mathbf{w}}(f)$-moderate (strictly $\mathbf{P}$-moderate) if and only if it is $\mathbf{P}_{\mathbf{w}}(f)$-moderate ( $\mathbf{P}$-moderate).

Lemma 2.24. A sequence $\left(a_{i}\right)_{i \in \omega}$ is $\mathbf{D}(f)$-moderate (D-moderate) if and only if there is a number $h$ such that $h f(i)^{n}>\left|a_{i}\right|+k$ (correspondingly, $h f(i)^{n}>\left|a_{i}\right|+k$ ) for all $i \in \mathbb{N}$.

Example 2.25. Given an increasing function $f(i)$, we can consider the set $\mathbf{E}(f)=\left\{e^{k f} ; k \in\right.$ $\left.\mathbb{R}^{++}\right\}$as the class $\mathbf{F}$. In particular, we have the set $\mathbf{E}=\left\{e^{k x} ; k \in \mathbb{R}^{++}\right\}$of positive exponents.

LEMma 2.26. A sequence $\left(a_{i}\right)_{i \in \omega}$ is strictly $\mathbf{E}(f)$-moderate (strictly $\mathbf{E}$-moderate) if and only if it is $\mathbf{E}(f)$-moderate (E-moderate).

Example 2.27. Given an increasing function $f(i)$, we can consider the $\operatorname{set} \mathbf{E}^{-1}(f)=\left\{e^{-k f}\right.$; $\left.k \in \mathbb{R}^{++}\right\}$as the class $\mathbf{F}$. In particular, we have the set $\mathbf{E}^{-1}=\left\{e^{-k x} ; k \in \mathbb{R}^{++}\right\}$of negative exponents.

Remark 2.28. The result of Lemma 2.26 is not true for $\mathbf{E}^{-1}(f)$-moderate sequences. As a result, the set $\mathbf{E}(f) \mathbb{R}^{\omega}$ of all $\mathbf{E}(f)$-moderate sequences (and in particular the set $\mathbf{E} \mathbb{R}^{\omega}$ ) is invariant with respect to hypernumbers, while the set $\mathbf{E}^{-1}(f) \mathbb{R}^{\omega}$ of all $\mathbf{E}^{-1}(f)$-moderate sequences (and in particular the set $\mathbf{E}^{-1} \mathbb{R}^{\omega}$ ) is not invariant.

In what follows, we assume that the class $\mathbf{F}$ of functions is closed from above with respect to addition and multiplication, that is, if $f, g \in \mathbf{F}$, then $\exists h \in \mathbf{F}(f+g \leq h)$ and $\exists v \in \mathbf{F}(f \cdot g \leq v)$.

Proposition 2.29. The classes $\mathbf{D}, \mathbf{E}$, and $\mathbf{E}^{-1}$ are closed from above with respect to addition and multiplication and the classes $\mathbf{D}(f), \mathbf{E}(f), \mathbf{E}^{-1}(f)$ are closed from above with respect to addition and multiplication when the function $f$ is strictly increasing.

Remark 2.30. The result of Proposition 2.29 is not true in general when the function $f$ is not strictly increasing.

Definition 2.31. For arbitrary sequences $a=\left(a_{i}\right)_{i \in \omega}, b=\left(b_{i}\right)_{i \in \omega} \in \mathbb{R}^{\omega}$

$$
a \sim_{\mathrm{F}} b \Longleftrightarrow \text { the sequence } c=\left(\left|a_{i}-b_{i}\right|\right)_{i \in \omega} \text { is strictly F-moderate. }
$$

LEMMA 2.32. The relation $\sim_{\mathrm{F}}$ is an equivalence.

Proof. The properties " $a \sim_{\mathrm{F}} a$ " and " $a \sim_{\mathrm{F}} b$ implies $b \sim_{\mathrm{F}} a$ " of an equivalence relation follow directly from the definition of the equivalence $\sim_{F}$, and we need to check only transitivity of this relation. 
Let $a \sim_{\mathrm{F}} b$ and $b \sim_{\mathrm{F}} c$. Then by the definition, there are numbers $k \in \mathbb{R}^{++}$and $m \in$ $\omega$ and a function $f \in \mathbf{F}$ such that for all $i>m$, we have $\left|a_{i}-b_{i}\right|+k<f(i)$, and there are numbers $l \in \mathbb{R}^{++}$and $n \in \omega$ and a function $g \in \mathbf{F}$ such that for all $i>n$, we have $\left|b_{i}-c_{i}\right|+l<g(i)$. Consequently, for $r=k+l$ and all $i>\max (m, n)$, we have $\left|a_{i}-c_{i}\right|+r \leq$ $\left.\left|a_{i}-b_{i}\right|+k+\left|b_{i}-c_{i}\right|+l<f(i)\right)+g(i) \leq h(i)$ for some function $h \in \mathbf{F}$ as the class $\mathbf{F}$ of functions is closed from above with respect to addition. It means that $a \sim_{\mathrm{F}} c$.

The lemma is proved.

This result allows us to define F-extended hypernumbers.

Definition 2.33. Classes of the equivalence $\sim_{\mathrm{F}}$ are called real $\mathbf{F}$-extended hypernumbers and their set is denoted by $\mathbf{F} \mathbb{R}_{\omega}$.

Any sequence $a=\left(a_{i}\right)_{i \in \omega}$ of real numbers determines an $\mathbf{F}$-extended real hypernumber $\alpha=\operatorname{Hn}^{\mathrm{F}}\left(a_{i}\right)_{i \in \omega}$. Real F-extended hypernumbers are sets of equivalent sequences of real numbers like rational numbers are sets of equivalent fractions or real number sets of equivalent fundamental sequences of rational numbers.

Theorem 2.34. FR $\mathbb{R}_{\omega}$ is a vector space over $\mathbb{R}$.

Let $\mathbf{F}$ be a set of functions from $\mathbb{R}^{++}$into $\mathbb{R}^{++}$and let $M \mathbf{F}$ be the closure of $\mathbf{F}$ with respect to addition and multiplication of functions and multiplication by elements from $\mathbb{R}^{++}$, that is, $M \mathbf{F}$ is the least linear algebra that contains $\mathbf{F}$.

Definition 2.35. The set $\mathbf{F}$ is called ordinal complete if for any element $p$ from $M \mathbf{F}$, there is an element $q$ from $\mathbf{F}$ larger than or equal to $p$.

Proposition 2.17 implies the following result.

Corollary 2.36. Any MF-moderate sequence $\left(a_{i}\right)_{i \in \omega}$ is $\mathbf{F}$-moderate.

Example 2.37. The sets $\mathbf{E}, \mathbf{E}^{-1}, \mathbf{P}, \mathbf{D}$ are ordinal complete.

In what follows, we take the set $\mathbf{E}^{-1}=\left\{e^{-k x} ; k \in \mathbb{R}^{++}\right\}$as the class $\mathbf{F}$ of functions and develop the theory for this case.

Definition 2.38. For arbitrary sequences $a=\left(a_{i}\right)_{i \in \omega}, b=\left(b_{i}\right)_{i \in \omega} \in \mathbb{R}^{\omega}$,

$$
a \sim_{\mathbf{E}} b \Longleftrightarrow \text { the sequence } c=\left(\left|a_{i}-b_{i}\right|\right)_{i \in \omega} \text { is } \mathbf{E}^{-1} \text {-moderate. }
$$

In other words, $a \sim_{\mathrm{E}} b$ if and only if there is $k \in \mathbb{R}^{++}$such that $\exists m \in \omega$ for all $i>m\left(\mid a_{i}-\right.$ $\left.b_{i} \mid<e^{-k i}\right)$.

LEMMA 2.39. The relation $\sim_{\mathrm{E}}$ is an equivalence.

Proof. Indeed, the properties “ $a \sim_{\mathrm{E}} a$ " and “ $a \sim_{\mathrm{E}} b$ implies $b \sim_{\mathrm{E}} a$ " follow directly from the definition, and we need to check only transitivity of this relation.

Let $a \sim_{\mathrm{E}} b$ and $b \sim_{\mathrm{E}} c$. Then by the definition, there are numbers $k \in \mathbb{R}^{++}$and $m \in \omega$ such that for all $i>m$, we have $\left|a_{i}-b_{i}\right|<e^{-k i}$ and there are numbers $h \in \mathbb{R}^{++}$and $n \in \omega$ such that for all $i>n$, we have $\left|b_{i}-c_{i}\right|<e^{-h i}$. Consequently, for all $i>\max (m, n)$, we have $\left|a_{i}-c_{i}\right| \leq\left|a_{i}-b_{i}\right|+\left|b_{i}-c_{i}\right|<e^{-k i}+e^{-h i}<e^{-l i}+e^{-l i}($ with $l=\min (k, h))=2 e^{-l i}<$ $e e^{-l i}=e^{1-k l i}<e^{(1 / q-l) i}=e^{-r i}$ with $r=l-1 / q$. It means that $a \sim_{\mathrm{E}} c$.

The lemma is proved. 
Definition 2.40. The classes of the equivalence $\sim_{\mathrm{E}}$ are called real $\mathbf{E}^{-1}$-extended hypernumbers, or simply $\mathbf{E}$-hypernumbers, and their set is denoted by $\mathbf{E} \mathbb{R}_{\omega}$.

Any sequence $a=\left(a_{i}\right)_{i \in \omega}$ determines an E-hypernumber $\alpha=\operatorname{Hn}_{\mathbf{E}}\left(a_{i}\right)_{i \in \omega}$. Real Ehypernumbers are sets of equivalent sequences of real numbers like rational numbers are sets of equivalent fractions or real number sets of equivalent fundamental sequences of rational numbers.

Remark 2.41. There are E-hypernumbers more than hypernumbers.

Lemma 2.42. The set $\mathbb{R}$ of all real numbers is isomorphically included into $\mathbf{E R}_{\omega}$.

Proposition 2.43. There are projections $\mathbb{R}^{\omega} \stackrel{p_{\mathrm{E}}}{\longrightarrow} \mathrm{ER}_{\omega} \stackrel{q_{\mathrm{E}}}{\longrightarrow} \mathbb{R}_{\omega}$.

Remark 2.44. In the set $\mathbf{E} \mathbb{R}_{\omega}$, there are numbers such that they are smaller than any positive real number and larger than zero. For example, $\alpha=\operatorname{Hn}_{\mathrm{E}}(1 / i)_{i \in \omega} \neq 0$ and for any $r \in \mathbb{R}^{++}, \alpha<r$.

Remark 2.45. It is possible in the same way as for ordinary sequences of real numbers to define E-hypernumbers for more general (than partially ordered) sets of indices, in particular, E-hypernumbers may be defined by $\omega^{2}$-sequences, that is, sets of real numbers indexed by elements from $\omega^{2}$. The sets of E-hypernumbers that are defined by different sets of indices do not coincide.

The relations on $\mathbb{R}$ induce corresponding relations on $\mathbb{R}^{\omega}$.

Definition 2.46. If $a, b \in \mathbb{R}^{\omega}$, then

$$
\begin{array}{ll}
a \leq b \Longleftrightarrow \exists n & \forall i \geq n\left(a_{i} \leq b_{i}\right), \\
a<b \Longleftrightarrow \exists n & \forall i \geq n\left(a_{i}<b_{i}\right) .
\end{array}
$$

Lемма 2.47. The relations $\leq$ and $<$ on $\mathbb{R}^{\omega}$ are a partial order and strict partial order, respectively.

These relations induce similar relations on $\mathbb{R}_{\omega}$.

Definition 2.48. If $\alpha, \beta \in \mathbf{E} \mathbb{R}_{\omega}$, then

$$
\begin{gathered}
\alpha \leq \beta \Longleftrightarrow \exists a \in \alpha, \exists b \in \beta \quad(a \leq b), \\
\alpha<\beta \Longleftrightarrow(\exists a \in \alpha, \exists b \in \beta(a<b)) \text { and } \alpha \neq \beta .
\end{gathered}
$$

LEMMA 2.49. The relations $\leq$ and $<$ on $\mathbf{E R}_{\omega}$ are a partial order and a strict partial order, respectively.

There are two operations in $\mathbb{R}^{++}$: addition and multiplication. These operations induce three operations in sets of functions from $\mathbb{R}^{++}$into $\mathbb{R}^{++}$: addition of functions, multiplication by elements from $\mathbb{R}^{++}$, and multiplication of functions.

Theorem 2.50. $\mathbf{E R}_{\omega}$ is a vector space over $\mathbb{R}$. 
Proof. To be a vector space over the algebra $\mathbb{R}$, the set $\mathbb{E}_{\omega}$ has to possess two operations: addition + and multiplication by elements from $\mathbb{R}$, which satisfy corresponding identities (Mallios [30]).

(1) Let $\alpha, \beta \in \mathbf{E} \mathbb{R}_{\omega}$. To define addition $\alpha+\beta=\gamma$ in $\mathbf{E R}_{\omega}$, we take some sequences $a=$ $\left(a_{i}\right)_{i \in \omega} \in \alpha$ and $b=\left(b_{i}\right)_{i \in \omega} \in \beta$ and determine the E-hypernumber $\gamma=\operatorname{Hn}_{\mathrm{E}}\left(a_{i}+b_{i}\right)_{i \in \omega}$. Indeed, $\left(a_{i}+b_{i}\right)_{i \in \omega}$ is a sequence in $\mathbb{R}^{\omega}$ and thus defines some $\mathbf{E}$-hypernumber. To show that this is a correct definition of an operation in $\mathbf{E} \mathbb{R}_{\omega}$, it is necessary to prove that $\gamma$ belongs to $\mathbb{E R}_{\omega}$ and does not depend on the choice of sequences $a$ and $b$. To prove the second statement, let us take another sequence $l=\left(l_{i}\right)_{i \in \omega}$ in $\beta$ and show that if the hypernumber $\delta$ is equal to $\operatorname{Hn}_{\mathbf{E}}\left(a_{i}+l_{i}\right)_{i \in \omega}$, then $\delta=\gamma$.

Indeed, $\left|\left(a_{i}+b_{i}\right)-\left(a_{i}+l_{i}\right)\right|=\left|b_{i}-l_{i}\right|$ for all $i$. By Definition 2.4, the sequence $c=$ $\left(\left|b_{i}-l_{i}\right|\right)_{i \in \omega}$ is $\mathbf{E}^{-1}$-moderate. Consequently, $\left(a_{i}+b_{i}\right)_{i \in \omega} \sim_{\mathbf{E}}\left(a_{i}+l_{i}\right)_{i \in \omega}$ and $\delta=\gamma$. If we take another sequence that represents the E-hypernumber $\alpha$, the result of addition will be the same E-hypernumber $\gamma$.

(2) Let $\alpha \in \mathbb{E R}_{\omega}$ and $c \in \mathbb{R}$. To define the product $c \alpha=\gamma$ in $\mathbb{E R}_{\omega}$, we take some sequence $a=\left(a_{i}\right)_{i \in \omega} \in \alpha$ and determine the E-hypernumber $\gamma=\operatorname{Hn}_{\mathbf{E}}\left(c a_{i}\right)_{i \in \omega}$. The proof that the product of an E-hypernumber and a real number is defined correctly is similar to the proof that the sum of two E-hypernumbers is defined correctly.

Necessary identities for operation in $\mathbf{E R}_{\omega}$ follow for the corresponding identities for multiplication and addition of real numbers.

The theorem is proved.

Lemma 2.51. The concept of a majorant from the class $\mathbf{D}$ is invariant with respect to the choice of the sequence that represents an $\mathbf{E}$-hypernumber.

Proof. By Lemma 2.13, it is sufficient to show that if $k x^{n}$ is a majorant of a sequence $a=$ $\left(a_{i}\right)_{i \in \omega}$ and $\operatorname{Hn}_{\mathbf{E}}\left(a_{i}\right)_{i \in \omega}=\operatorname{Hn}_{\mathbf{E}}\left(b_{i}\right)_{i \in \omega}$, then for some number $h \in \mathbb{R}^{++}, h x^{n}$ is a majorant of a sequence $b=\left(b_{i}\right)_{i \in \omega}$. Indeed, the equivalence $a \sim_{\mathrm{E}} b$ means (cf. Definition 2.4) that there are $m \in \mathbb{N}$ and $q \in \mathbb{R}^{++}$such that $\left|a_{i}-b_{i}\right|<e^{-q i}$ when $i>m$. In addition, $\exists t \in \omega$ for all $i>t\left(\left|a_{i}\right|<k i^{n}\right)$. Then $\left|b_{i}\right|=\left|b_{i}-a_{i}+a_{i}\right| \leq\left|b_{i}-a_{i}\right|+\left|a_{i}\right|<e^{-q i}+k i^{n}$ when $i>$ $\max \{m, t\}$. At the same time, $e^{-q i}<1<i^{n}$. So, $\left|b_{i}\right|<(1+k) i^{n}$ and $h x^{n}$ is a majorant for $b$ with $h=1+k$.

The lemma is proved.

Corollary 2.52. If a sequence $a=\left(a_{i}\right)_{i \in \omega}$ is $\mathbf{D}$-moderate and $a \sim_{\mathrm{E}} b$, then the sequence $b$ is also D-moderate.

This allows us to introduce D-moderate E-hypernumbers.

Definition 2.53. An E-hypernumber $\alpha=\operatorname{Hn}_{\mathbf{E}}\left(a_{i}\right)_{i \in \omega}$ is called D-moderate if some function $f(i)$ from $\mathbf{D}$ is its majorant.

The set of all D-moderate real E-hypernumbers is denoted by $\mathbf{E}^{D} \mathbb{R}_{\omega}$.

Proposition 2.54. $\mathbf{E}^{\mathbf{D}} \mathbb{R}_{\omega}$ is a linear subspace of $\mathbf{E R}_{\omega}$.

Proof. (a) If $\alpha=\operatorname{Hn}_{\mathbf{E}}\left(a_{i}\right)_{i \in \omega}, \beta=\operatorname{Hn}_{\mathbf{E}}\left(b_{i}\right)_{i \in \omega} \in \mathbf{E}^{\mathbf{D}} \mathbb{R}_{\omega}$, and $\gamma=\alpha+\beta$ in $\mathbf{E} \mathbb{R}_{\omega}$, then Ehypernumber $\gamma=\operatorname{Hn}_{\mathbf{E}}\left(c_{i}\right)_{i \in \omega}$, where $c_{i}=a_{i}+b_{i}$ for all $i \in \omega$. As $\alpha$ and $\beta$ are $\mathbf{D}$-moderate E-hypernumbers, there are numbers $k, h, m, n \in \mathbb{R}^{++}$such that $k x^{n}$ is a majorant of 
a sequence $a=\left(a_{i}\right)_{i \in \omega}$ and $h x^{m}$ is a majorant of a sequence $b=\left(b_{i}\right)_{i \in \omega}$, that is, $\left|a_{i}\right|<k i^{n}$ and $\left|b_{i}\right|<h i^{m}$ for all $i \in \omega$. Then $\left|c_{i}\right|=\left|a_{i}+b_{i}\right| \leq\left|a_{i}\right|+\left|b_{i}\right|<k i^{n}+h i^{m} \leq k i^{q}+h i^{q}=$ $(k+h) i^{q}$ for $q=\max \{m, n\}$. It means that $\gamma$ is also a $\mathbf{D}$-moderate $\mathbf{E}$-hypernumber.

(b) Let $\alpha=\operatorname{Hn}_{\mathbf{E}}\left(a_{i}\right)_{i \in \omega} \in \mathbf{E R}_{\omega}, c \in \mathbb{R}$, and $c \alpha=\theta$ in $\mathbf{E R}_{\omega}$. Then we have the Ehypernumber $\theta=\operatorname{Hn}_{\mathbb{E}}\left(c a_{i}\right)_{i \in \omega}$. Consequently, $\left|c \cdot a_{i}\right| \leq|c| \cdot\left|a_{i}\right|<|c| \cdot k i^{n}=h i^{n}$ for $h=$ $|c| \cdot k$. It means that $\theta$ is also a $\mathbf{D}$-moderate $\mathbf{E}$-hypernumber.

The proposition is proved.

Theorem 2.55. $\mathbf{E}^{\mathbf{D}} \mathbb{R}_{\omega}$ is a linear algebra over $\mathbb{R}$.

Proof. To be a linear algebra over the algebra $\mathbb{R}$, the set $\mathbf{E}^{\mathbf{D}} \mathbb{R}_{\omega}$ has to possess three operations: addition + , multiplication ', and multiplication by elements from $\mathbb{R}$. Two of these operations are defined in Theorem 2.34 and we need only to determine multiplication of D-moderate E-hypernumbers.

Let $\alpha, \beta \in \mathbf{E}^{\mathbf{D}} \mathbb{R}_{\omega}$. To define multiplication $\alpha \cdot \beta=\gamma$ in $\mathbf{E}^{\mathbf{D}} \mathbb{R}_{\omega}$, we take some sequences $a=\left(a_{i}\right)_{i \in \omega} \in \alpha$ and $b=\left(b_{i}\right)_{i \in \omega} \in \beta$ and determine the E-hypernumber $\gamma=\operatorname{Hn}_{\mathbf{E}}\left(a_{i} \cdot b_{i}\right)_{i \in \omega}$. Indeed, $\left(a_{i} \cdot b_{i}\right)_{i \in \omega}$ is a sequence in $\mathbb{R}^{\omega}$ and thus defines some $\mathbf{E}$-hypernumber. To show that this defines an operation in $\mathbf{E}^{\mathbf{D}} \mathbb{R}_{\omega}$, it is necessary to prove that $\gamma$ belongs to $\mathbf{E}^{\mathbf{D}} \mathbb{R}_{\omega}$ and does not depend on the choice of sequences $a$ and $b$.

By the definition of $\mathbf{D}$-moderate E-hypernumbers, there are numbers $k, h, m, n \in \mathbb{R}^{++}$ such that $\left|a_{i}\right|<k i^{n}$ and $\left|b_{i}\right|<h i^{m}$ for all $i \in \omega$. Then $\left|a_{i} \cdot b_{i}\right| \leq\left|a_{i}\right| \cdot\left|b_{i}\right|<k i^{n} \cdot h i^{m}=$ $(k h) i^{n+m}$ for all $i \in \omega$. It means that $\gamma$ is a $\mathbf{D}$-moderate $\mathbf{E}$-hypernumber.

To prove the second statement, let us take another sequence $l=\left(l_{i}\right)_{i \in \omega}$ in $\beta$ and show that if the E-hypernumber $\delta$ is equal to $\operatorname{Hn}_{\mathbf{E}}\left(a_{i} \cdot l_{i}\right)_{i \in \omega}$, then $\delta=\gamma$.

Indeed, $\left|\left(a_{i} \cdot b_{i}\right)-\left(a_{i} \cdot l_{i}\right)\right|=\left|a_{i} \cdot\left(b_{i}-l_{i}\right)\right|$ (as multiplication of real numbers is distributive) $\leq\left|a_{i}\right| \cdot\left|b_{i}-l_{i}\right|$ (by properties of the norm of real numbers) $<k i^{n} \cdot\left|b_{i}-l_{i}\right|<$ $k i^{n} \cdot e^{-q i}$. Then taking $r=1 / 2 q$, we have $k i^{n} \cdot e^{-q i}=e^{-r i}\left(k i^{n} / e^{r i}\right) \leq e^{-r i}$ as $k i^{n} / e^{r i}<1$ for sufficiently big $i \in \omega$. By Definition 2.4, the sequence $c=\left(\left|a_{i} \cdot b_{i}-a_{i} \cdot l_{i}\right|\right)_{i \in \omega}$ is $\mathbf{E}^{-1}$ moderate. Consequently, $\left(a_{i} \cdot b_{i}\right)_{i \in \omega} \sim_{\mathbf{E}}\left(a_{i} \cdot l_{i}\right)_{i \in \omega}$ and $\delta=\gamma$. If we take another sequence that represents the E-hypernumber $\alpha$, the result of multiplication will be the same $\mathbf{E}$ hypernumber $\gamma$.

To conclude, we need to prove distributivity of multiplication with respect to addition. Let us consider two sequences $\left(a_{i} \cdot\left(b_{i}+c_{i}\right)\right)_{i \in \omega}$ and $\left(a_{i} \cdot b_{i}+a_{i} \cdot c_{i}\right)_{i \in \omega}$. Taking the absolute value of their differences, we have $\left(\left|\left(a_{i} \cdot\left(b_{i}+c_{i}\right)\right)_{i \in \omega}-\left(a_{i} \cdot b_{i}+a_{i} \cdot c_{i}\right)\right|\right)_{i \in \omega}=\left(\mid\left(a_{i} \cdot\left(b_{i}+\right.\right.\right.$ $\left.\left.\left.c_{i}\right)\right)-\left(a_{i} \cdot b_{i}+a_{i} \cdot c_{i}\right) \mid\right)_{i \in \omega}=(0)=0$ as distributivity law is valid for real numbers. Thus, for any $\mathbf{D}$-moderate $\mathbf{E}$-hypernumbers $\alpha, \beta$, and $\gamma$, we have $\alpha \cdot(\beta+\gamma)=\alpha \cdot \beta+\alpha \cdot \gamma$.

The theorem is proved.

\section{Topology in the space of E-hypernumbers}

Here we consider the set $\mathbf{E} \mathbb{R}_{\omega}$ as a topological space. It is possible to define various topologies in $\mathbf{E R}_{\omega}$. As $\mathbf{E} \mathbb{R}_{\omega}$ is quotient set of the set $\mathbb{R}^{\omega}$ of all sequences of natural numbers, we can consider the topology $\tau_{s p}$ on $\mathbb{R}^{\omega}$ determined by the system $T$ of all spherical neighborhoods (Burgin [7]) and take the topology $\delta_{s p}$ induced in $\mathbf{E} \mathbb{R}_{\omega}$ by $\tau_{s p}$. On $\mathbb{R}$ as a subset of $\mathbb{R}^{\omega}, \tau_{s p}$ induces the natural topology for real numbers. 
However, results from Burgin [4] demonstrate that $\delta_{s p}$ is not a Hausdorff topology. Thus, to get a good topology in $\mathbb{E R}_{\omega}$, we need to start with another topology in $\mathbb{R}^{\omega}$.

Definition 3.1. Two elements $a=\left(a_{i}\right)_{i \in \omega}$ and $b=\left(b_{i}\right)_{i \in \omega} \in \mathbb{R}^{\omega}$ are called similar (denoted $a \approx b)$ if there is $n \in \omega$ such that for all $i>n,\left(a_{i}=b_{i}\right)$.

Proposition 3.2. . Similarity $\approx$ is an equivalence relation and defines a closure operation on subsets of $\mathbb{R}^{\omega}$.

Proof. If $A$ is a subset of $\mathbb{R}^{\omega}$, we define $\mathrm{Cl}_{\text {sim }}(A)=\left\{d \in \mathbb{R}^{\omega} ; \exists a \in A \wedge d \approx a\right\}$. This construction satisfies all axioms of a closure operation (cf. Kuratowski [25]). Indeed, by the definition, $\mathrm{Cl}_{\text {sim }}(A \cup B)=\mathrm{Cl}_{\text {sim }}(A) \cup \mathrm{Cl}_{\text {sim }}(B), A \subseteq \mathrm{Cl}_{\text {sim }}(A), \mathrm{Cl}_{\text {sim }}(\varnothing)=\varnothing$, and $\mathrm{Cl}_{\text {sim }}\left(\mathrm{Cl}_{\text {sim }}(A)\right)=\mathrm{Cl}_{\text {sim }}(A)$.

Definition 3.1 implies reflexivity, symmetry, and transitivity of the relation $\approx$, that is, $\approx$ is an equivalence relation.

Let us take a set $\mathbf{H}$ of functions $f: \mathbb{R}^{++} \rightarrow \mathbb{R}^{++}$. It is possible to define a specific topology in the space $\mathbb{R}^{\omega}$ by means of neighborhoods that depend on functions from $\mathbf{H}$. We call it the $\mathbf{H}$-conical topology in the space $\mathbb{R}^{\omega}$.

Definition 3.3. An $\mathbf{H}$-conical neighborhood of a sequence $a \in \mathbb{R}^{\omega}$ is a set $O a \subseteq \mathbb{R}^{\omega}$ that satisfies the following conditions:

(1) $c=\left(c_{i}\right)_{i \in \omega} \in O a \Rightarrow a-|c-a| \in O a$ and $a+|c-a| \in O a$;

(2) $c=\left(c_{i}\right)_{i \in \omega} \in O a \wedge c \approx b \Rightarrow b \in O a$;

(3) for all $d=\left(d_{i}\right)_{i \in \omega} \in \mathbb{R}^{\omega},\left(c=\left(c_{i}\right)_{i \in \omega} \in O a\right.$ and there is $n \in \omega$ such that for all $i>$ $\left.n,\left(\left|a_{i}-d_{i}\right| \leq\left|a_{i}-c_{i}\right|\right) \Rightarrow d \in O a\right)$

(4) for all $d=\left(d_{i}\right)_{i \in \omega} \in O a,\left(\exists b=\left(b_{i}\right)_{i \in \omega} \in O a(b>d)\right.$ and $\left.\exists c=\left(c_{i}\right)_{i \in \omega} \in O a(c<d)\right)$;

(5) for all $f \in \mathbf{H},\left(\left(a_{i} \pm f^{-1}(i)\right)_{i \in \omega} \in O a\right)$.

It is possible to define the $\mathbf{H}$-conical neighborhood $\mathrm{Oa}$ in a different way, changing the fifth condition.

LEMMA 3.4. The following conditions are equivalent if condition (4) is valid:

(5) for all $f \in \mathbf{H},\left(\left(a_{i} \pm f^{-1}(i)\right)_{i \in \omega} \in O a\right)$,

$\left(5^{\circ}\right)$ for all $f \in \mathbf{H}$, there is $n \in \omega$ such that for all $\left.i>n \exists c=\left(c_{i}\right)_{i \in \omega} \in O a\left(c_{i}-a_{i}>f^{-1}(i)\right)\right)$ and there is $m \in \omega$ such that for all $i>m \exists d=\left(d_{i}\right)_{i \in \omega} \in O a\left(a_{i}-d_{i}>f^{-1}(i)\right)$.

Example 3.5. The set $O_{1 / x} \mathbf{0}=\left\{c=\left(c_{i}\right)_{i \in \omega} \in \mathbb{R}^{\omega} ; \exists n \in \omega\right.$ for all $\left.i>n\left(\left|c_{i}\right|<1 / i\right)\right\}$ is an Econical neighborhood of the sequence $\mathbf{0}=\left(a_{i}=0\right)_{i \in \omega} \in \mathbb{R}^{\omega}$.

Example 3.6. Let $a=\mathbf{i}=\left(a_{i}=1 / i\right)_{i \in \omega}$ and $b=\mathbf{i}^{2}=\left(b_{i}=1 / i^{2}\right)_{i \in \omega}$. The set $O a=\{c=$ $\left.\left(c_{i}\right)_{i \in \omega} \in \mathbb{R}^{\omega} ;|c-a|<\mathbf{i}^{2}\right\}$ is an E-conical neighborhood of the sequence $a$. Indeed, if we take an element $d=a+|c-a| \in \mathbb{R}^{\omega}$, then $|d-a|=|a+| c-a|-a|=|c-a|<\mathbf{i}^{2}$. Thus, $d=a+|c-a| \in O a$ and a similar reasoning gives us that $e=a-|c-a| \in O a$. Consequently, condition (1) from Definition 3.3 is valid for the set $O a$. Condition (2) directly follows from Definition 3.1 and the construction of $\mathrm{Oa}$. Condition (3) directly follows from the construction of $\mathrm{Oa}$.

Let $c=\left(c_{i}\right)_{i \in \omega} \in \mathbb{R}^{\omega}$ and $|c-a|<\mathbf{i}^{2}$. Then for $d=\left(d_{i}\right)_{i \in \omega} \in \mathbb{R}^{\omega}$ with $d_{i}=c_{i}+(1 / 2)$ $\left(1 / i^{2}-\left|c_{i}-a_{i}\right|\right)$, we have $d>c$ and $d \in O a$. In a similar way, for $b=\left(b_{i}\right)_{i \in \omega} \in \mathbb{R}^{\omega}$ with $b_{i}=c_{i}-(1 / 2)\left(1 / i^{2}-\left|c_{i}-a_{i}\right|\right)$, we have $b<c$ and $b \in O a$. This implies condition (4). 
Let us check condition (5). For E-conical neighborhoods, it has the following form: for all $k \in \mathbb{R}^{++},\left(a \pm \mathbf{e}_{k}=\left(a_{i}\right)_{i \in \omega} \pm\left(e^{-k i}\right)_{i \in \omega}=\left(a_{i} \pm e^{-k i}\right)_{i \in \omega} \in O a\right)$. To show that given a sequence $\mathbf{e}_{k}=\left(e^{-k i}\right)_{i \in \omega}$ from $\mathbb{R}^{\omega}$, we have $a+\mathbf{e}_{k} \in O a$ and $a-\mathbf{e}_{k} \in O a$, we consider the inequality $a_{i}+e^{-k i}<a_{i}+1 / i^{2}$. It is equivalent to $i^{2}<e^{k i}$. Taking the Taylor series for $e^{k i}$ (cf., e.g., (Ross, [42])), we have $(k i)^{3} / 6<1+k i+(k i)^{2} / 2+(k i)^{3} / 6<e^{k i}$. Then $i^{2}<(k i)^{3} / 6$ if $i>\left[6 / k^{3}\right]+1$. Consequently, $a+\mathbf{e}_{k} \in O a$ as $a+i^{2}<a+e^{k i}$ when $i>\left[6 / k^{3}\right]+1$. Condition (1) implies that for the same sequence $\mathbf{e}_{k}$, we have $a-\mathbf{e}_{k} \in O a$. As $e^{k i}$ is an arbitrary function from $\mathbf{E}$, condition (5) is also valid for the set $\mathrm{Oa}$, that is, it is an E-conical neighborhood of the sequence $a$.

Condition (3) for $\mathbf{H}$-conical neighborhood implies the following results.

LEMma 3.7. If $\mathrm{O} \mathbf{0}$ is an $\mathbf{H}$-conical neighborhood of the sequence $\mathbf{0} \in \mathbb{R}^{\omega}$ and $c=\left(c_{i}\right)_{i \in \omega} \in$ O0, then $|c|=\left(\left|c_{i}\right|\right)_{i \in \omega} \in O 0$.

Lemma 3.8. If $\mathrm{Oa}$ is an $\mathbf{H}$-conical neighborhood of a sequence $a \in \mathbb{R}^{\omega}, c=\left(c_{i}\right)_{i \in \omega} \in \mathbb{R}^{\omega}$, and $a+|c-a| \in O a$, then $c \in O a$.

Indeed, for all $i \in \omega,\left(\left|a_{i}-c_{i}\right| \leq\left|a_{i}-\left(a_{i}+\left|c_{i}-a_{i}\right|\right)\right|=\left|a_{i}-c_{i}\right|\right)$, and by condition (3), $c \in O a$.

Proposition 3.9. The system $T$ of all $\mathbf{H}$-conical neighborhoods determines a topology $\tau_{c \mathbf{H}}$ on $\mathbb{R}^{\omega}$.

Proof. Let us define open sets in $\mathbb{R}^{\omega}$ by the following rule: a set $X$ in $\mathbb{R}^{\omega}$ is open if for any point $x$ from $X$, it contains some $\mathbf{H}$-conical neighborhood of $x$. To prove that we have a topology, we demonstrate that the set of all open sets is closed with respect to arbitrary unions and finite intersections. For unions, this property follows directly from the definition. To prove this property for intersections, it is sufficient to show that the intersection of two arbitrary $\mathbf{H}$-conical neighborhoods of a sequence $a \in \mathbb{R}^{\omega}$ is an $\mathbf{H}$-conical neighborhood of the same sequence $a$ (Kuratowski [25]). $\mathbb{R}^{\omega}$ is a vector space. Thus, by the definition of $\mathbf{H}$-conical neighborhoods, any $\mathbf{H}$-conical neighborhood $\mathrm{Oa}$ of a sequence $a \in \mathbb{R}^{\omega}$ is a shift of some $\mathbf{H}$-conical neighborhood $\mathrm{O} \mathbf{0}$ of $\mathbf{0}$, that is, $\mathrm{O} a=\mathrm{O} \mathbf{0}+a$. Consequently, to prove our assertion, we need to consider only $\mathbf{H}$-conical neighborhoods of $\mathbf{0}$.

Let us take the intersection $O \mathbf{0}=O_{1} \mathbf{0} \cap O_{2} \mathbf{0}$ of two $\mathbf{H}$-conical neighborhoods $O_{1} \mathbf{0}$ and $\mathrm{O}_{2} \mathbf{0}$ of the element $\mathbf{0}=(0)_{i \in \omega} \in \mathbb{R}^{\omega}$ and show that this set is an $\mathbf{H}$-conical neighborhood of $\mathbf{0}$. To do this, we need to check conditions (1)-(5) from Definition 3.3.

Condition 1. Let $c=\left(c_{i}\right)_{i \in \omega} \in O \mathbf{0}$. Then $c \in O_{1} \mathbf{0}$. Consequently, by condition (1), we have $a-|c-a| \in O_{1} \mathbf{0}$ and $a+|c-a| \in O_{1} \mathbf{0}$. In a similar way, $a-|c-a| \in O_{2} \mathbf{0}$ and $a+\mid c-$ $a \mid \in \mathrm{O}_{2}$ 0. Thus, $a-|c-a| \in O \mathbf{0}$ and $a+|c-a| \in O \mathbf{0}$, that is, condition (1) is true for O0.

Condition 2. If $c=\left(c_{i}\right)_{i \in \omega} \in O \mathbf{0}$ and $c \approx b$, then $c \in O_{1} \mathbf{0}$ and $b \in O_{1} \mathbf{0}$. At the same time, $c \in \mathrm{O}_{2} \mathbf{0}$ and $b \in \mathrm{O}_{2} \mathbf{0}$ as $\mathrm{O}_{1} \mathbf{0}$ and $\mathrm{O}_{2} \mathbf{0}$ are $\mathbf{H}$-conical neighborhoods of $\mathbf{0}$. Consequently, $b \in O \mathbf{0}=O_{1} \mathbf{0} \cap \mathrm{O}_{2} \mathbf{0}$, that is, condition (2) is true for $O \mathbf{0}$.

Condition 3. Let $d=\left(d_{i}\right)_{i \in \omega} \in \mathbb{R}^{\omega}$ and for some $c=\left(c_{i}\right)_{i \in \omega}$ from $O \mathbf{0}$, the following condition is true: there is $n \in \omega$ such that for all $i>n,\left(\left|a_{i}-d_{i}\right| \leq\left|a_{i}-c_{i}\right|\right)$. Then $d \in O \mathbf{0}=$ $\mathrm{O}_{1} \mathbf{0} \cap \mathrm{O}_{2} \mathbf{0}$ because $c \in O \mathbf{0}=\mathrm{O}_{1} \mathbf{0} \cap \mathrm{O}_{2} \mathbf{0}$. Consequently, condition (3) is true for $O \mathbf{0}$. 
Condition 4. Let $d=\left(d_{i}\right)_{i \in \omega} \in O \mathbf{0}$. Then there is $b=\left(b_{i}\right)_{i \in \omega} \in O_{1} \mathbf{0}$ such that $b>d$ and there is $c=\left(c_{i}\right)_{i \in \omega} \in O_{2} \mathbf{0}$ such that $c>d$. Let us take the element $u=\left(u_{i}\right)_{i \in \omega}$, where $u_{i}=$ $\min \left\{d_{i}, c_{i}\right\}$ for all $i=1,2, \ldots$. Then, by definition, $u>d$ and $u \in O \mathbf{0}=O_{1} \mathbf{0} \cap O_{2} \mathbf{0}$ due to condition (3), which is true for both $\mathrm{O}_{1} \mathbf{0}$ and $\mathrm{O}_{2} \mathbf{0}$. In a similar way, we find an element $v=\left(v_{i}\right)_{i \in \omega}$ such that $v<d$ and $v \in O \mathbf{0}$. Consequently, condition (4) is true for $O \mathbf{0 .}$

Condition 5. If $f \in \mathbf{H}$ and $\left(a_{i} \pm f^{-1}(i)\right)_{i \in \omega} \in O_{1} \mathbf{0}$ and $\left(a_{i} \pm f^{-1}(i)\right)_{i \in \omega} \in O_{2} \mathbf{0}$, then $\left(a_{i} \pm\right.$ $\left.f^{-1}(i)\right)_{i \in \omega} \in O \mathbf{0}$, that is, condition (5) is true for $O \mathbf{0}$.

The proposition is proved as the whole set $\mathbb{R}^{\omega}$ and the empty set $\varnothing$ are open in this topology.

In what follows, we consider only $\mathbf{H}=\mathbf{E}$ and topology $\tau_{c \mathrm{E}}$.

As any spherical neighborhood is at the same time an E-conical neighborhood, we have the following result.

Proposition 3.10. Topology $\tau_{c \mathrm{E}}$ in $\mathbb{R}^{\omega}$ is stronger than topology $\tau_{s p}$.

Indeed, if we take an arbitrary element $\boldsymbol{a}$ from $\mathbb{R}^{\omega}$, then any spherical neighborhood of it contains some E-conical neighborhood, for example, a neighborhood of the type $O_{1 / x} \boldsymbol{a}$. Thus, any open set in the topology $\tau_{s p}$ will be open in the topology $\tau_{c \mathrm{E}}$.

Remark 3.11. In contrast to topology $\tau_{s p}$, topology $\tau_{c \mathbf{H}}$ in $\mathbb{R}^{\omega}$ does not induce the natural topology for real numbers $\mathbb{R}$ as a subset of $\mathbb{R}^{\omega}$. In topology $\tau_{c \mathrm{E}}$, the natural inclusion of $\mathbb{R}$ in $\mathbb{R}^{\omega}$ has discrete topology.

Indeed, $\mathbb{R}$ is included in $\mathbb{R}^{\omega}$ by the following correspondence: if $a \in \mathbb{R}$, then the correspondence $a \rightarrow \boldsymbol{a}=\left(a_{i}=a\right)_{i \in \omega} \in \mathbb{R}^{\omega}$ determines a natural inclusion of $\mathbb{R}$ into $\mathbb{R}^{\omega}$, that is, $\mathbb{R}$ is isomorphic to $\mathbb{R}_{\mathbf{o}} \subseteq \mathbb{R}^{\omega}$. Due to this inclusion, open sets in $\mathbb{R}_{\mathbf{o}}$ are intersections of open sets in $\mathbb{R}^{\omega}$ with $\mathbb{R}_{\mathbf{o}}$. If we take a neighborhood $O_{1 / x} \boldsymbol{a}$ of an element $a \in \mathbb{R}$, its intersection with $\mathbb{R}_{\mathbf{o}}$ consists of the single element $a$. Consequently, the set $\{a\}$ is open in $\mathbb{R}_{\mathbf{o}}$, and as the union of any number of open sets in a topological space is open, the set $\{a\}$ is closed. This means that $\mathbb{R}_{\mathbf{o}}$ has discrete topology.

Definition 3.12. If $r \in \mathbb{R}^{++}$, then $r \cdot \mathrm{O} a=\mathrm{Cl}_{\mathrm{sim}}\left(\left\{d=\left(d_{i}\right)_{i \in \omega} ; d=r \cdot c=\left(r c_{i}\right)_{i \in \omega} ; c=\right.\right.$ $\left.\left.\left(c_{i}\right)_{i \in \omega} \in O a\right\}\right)$.

Example 3.13. Let $r \in \mathbb{R}^{++}, a=\mathbf{i}=\left(a_{i}=1 / i\right)_{i \in \omega}$, and $b=\mathbf{i}^{2}=\left(b_{i}=1 / i^{2}\right)_{i \in \omega}$. The set $O a=$ $\left.\left\{c=\left(c_{i}\right)_{i \in \omega} \in \mathbb{R}^{\omega} ;|c-a|<\mathbf{i}^{2}\right)\right\}$ is an E-conical neighborhood of the sequence $a$ and $r$. $O a=\left\{c=\left(c_{i}\right)_{i \in \omega} \in \mathbb{R}^{\omega} ;|c-r \cdot a|<r \cdot \mathbf{i}^{2}\right\}=\left\{c=\left(c_{i}\right)_{i \in \omega} \in \mathbb{R}^{\omega} ; r^{-1} \cdot|c-r \cdot a|<\mathbf{i}^{2}\right\}$.

LEMMA 3.14. If Oa is an $\mathbf{E}$-conical neighborhood of a sequence $a \in \mathbb{R}^{\omega}$, then for any $r \in \mathbb{R}^{++}$, $r \cdot$ Oa is an $\mathbf{E}$-conical neighborhood of the sequence $r \cdot a \in \mathbb{R}^{\omega}$.

Proof. As $\mathrm{O} a=\mathrm{O} 0+a$ and $r \cdot \mathrm{O} a=r \cdot \mathrm{O} \mathbf{0}+r \cdot a$, we can consider only E-conical neighborhoods of $\mathbf{0}$. To show that $r \cdot \mathrm{O} \mathbf{0}$ is an $\mathbf{E}$-conical neighborhood of $\mathbf{0}$, we need to check conditions (1)-(5) from Definition 3.3.

Condition 1. Let $c=\left(c_{i}\right)_{i \in \omega} \in r \cdot O \mathbf{0}$. Then $c \approx r \cdot b$ for some $b=\left(b_{i}\right)_{i \in \omega} \in O \mathbf{0}$. By condition (1), we have $|b| \in O 0$ and $-|b| \in O 0$. Thus, $|r \cdot b|=r \cdot|b| \in r \cdot O \mathbf{0}$ and $-|r \cdot b|=$ $-r \cdot|b|=r \cdot(-|b|) \in r \cdot$ O0. At the same time, $c \approx r \cdot b$ implies $|c| \approx|r \cdot b|$. As the set 
$r \cdot \mathrm{O} 0$ is closed with respect to similar elements, we have $|c| \in r \cdot \mathrm{O} \mathbf{0}$ and $-|c| \in r \cdot \mathrm{O} \mathbf{0}$, that is, condition (1) is true for $r \cdot O \mathbf{O}$.

Condition 2. This condition is true for $r \cdot \mathrm{O} 0$ because similarity closure is an idempotent operation (Kuratowski [25, Axiom 4]).

Condition 3. Let $d=\left(d_{i}\right)_{i \in \omega} \in \mathbb{R}^{\omega}$, and for some $c=\left(c_{i}\right)_{i \in \omega}$ from $r \cdot O 0$, the following condition is true: there is $n \in \omega$ such that for all $i>n,\left(\left|d_{i}\right| \leq\left|c_{i}\right|\right)$. Then for the same $n \in \omega$, we have $r^{-1} \cdot\left|d_{i}\right| \leq r^{-1} \cdot\left|c_{i}\right|$ when $i>n$. By condition (2) for $O \mathbf{0}, r^{-1} \cdot|c| \in O \mathbf{0}$. Then by condition (3) for $O \mathbf{0}, r^{-1} \cdot|d| \in O \mathbf{0}$. Consequently, $|d|=r \cdot\left(r^{-1} \cdot|d|\right) \in r \cdot O \mathbf{0}$. Lemma 3.8 implies that $d \in O \mathbf{0}$, that is, condition (3) is true for $r \cdot O \mathbf{0}$.

Condition 4. Let $d=\left(d_{i}\right)_{i \in \omega} \in r \cdot$ O0. Then $d \approx r \cdot b$ for some $b=\left(b_{i}\right)_{i \in \omega} \in O 0$. Then there is $a=\left(a_{i}\right)_{i \in \omega} \in O 0$ such that $b>a$ and there is $c=\left(c_{i}\right)_{i \in \omega} \in O 0$ such that $c>b$. As $r \in \mathbb{R}^{++}, r \cdot b>r \cdot a$ and $r \cdot c>r \cdot b$. Then $d>r \cdot a$ and $r \cdot c>d$. Besides, $r \cdot a$ and $r \cdot c$ belong to $r \cdot \mathrm{O0}$. It means that condition (4) is true for $r \cdot \mathrm{O}$.

Condition 5. Let us consider an arbitrary function $e^{-h i}$ from $\mathbf{E}^{-1}$ and the sequence $\mathbf{e}^{-h i}=$ $\left(e^{-h i}\right)_{i \in \omega}$. By the definition, $\mathbf{e}^{-(h+1) i}=\left(e^{-(h+1) i}\right)_{i \in \omega} \in O \mathbf{0}$. Then $\mathbf{e}^{-h i}<r \cdot \mathbf{e}^{-(h+1) i} \in r \cdot O \mathbf{0}$ and by condition (3), which has been already proved, $\mathbf{e}^{-h i} \in r \cdot O \mathbf{0}$, that is, condition (5) is true for $O \mathbf{0}$.

The lemma is proved.

Definition 3.15. If $r \in \mathbb{R}^{++}$, then $r \circ O a=\mathrm{Cl}_{\text {sim }}\left(\left\{d=\left(d_{i}\right)_{i \in \omega} ; d=\left(a_{i}-r\left(a_{i}-c_{i}\right)\right)_{i \in \omega} ; c=\right.\right.$ $\left.\left.\left(c_{i}\right)_{i \in \omega} \in O a\right\}\right)$.

Example 3.16. Let $r \in \mathbb{R}^{++}, a=\mathbf{i}=\left(a_{i}=1 / i\right)_{i \in \omega}$, and $b=\mathbf{i}^{2}=\left(b_{i}=1 / i^{2}\right)_{i \in \omega}$. The set $O a=$ $\left\{c=\left(c_{i}\right)_{i \in \omega} \in \mathbb{R}^{\omega} ;|c-a|<\mathbf{i}^{2}\right\}$ is an E-conical neighborhood of the sequence $a$ and $r \circ$ $\mathrm{O} a=\left\{c=\left(c_{i}\right)_{i \in \omega} \in \mathbb{R}^{\omega} ;|c-a|<r \cdot \mathbf{i}^{2}\right\}=\left\{c=\left(c_{i}\right)_{i \in \omega} \in \mathbb{R}^{\omega} ; r^{-1} \cdot|c-a|<\mathbf{i}^{2}\right\}$.

Lemma 3.17. If Oa is an $\mathbf{E}$-conical neighborhood of a sequence $a \in \mathbb{R}^{\omega}$, then for any $r \in \mathbb{R}^{++}$, $r \circ \mathrm{O} a$ is an $\mathbf{E}$-conical neighborhood of the sequence $a \in \mathbb{R}^{\omega}$.

Proof. As in Lemma 3.14, we can consider only E-conical neighborhoods of $\mathbf{0}$. However, for any such neighborhood $\mathrm{O} 0$, we have $r \circ \mathrm{O} 0=r \cdot \mathrm{O}$. Thus, Lemma 3.17 follows from Lemma 3.14.

A topological space $X$ may satisfy the following axioms (Kelley [24]).

$\left(T_{0}\right)$ (The Kolmogorov axiom). For all $x, y \in X,(\exists O x(y \notin O x) \vee \exists O y(x \notin O y))$.

$\left(T_{1}\right)$ (The Alexandroff axiom). For all $x, y \in X, \exists O x, \exists O y(x \notin O y \& y \notin O x)$.

$\left(T_{2}\right)$ (The Hausdorff axiom). For all $x, y \in X, \exists O x, \exists O y(O x \cap O y=\varnothing)$.

Here $O x, O y$ are neighborhoods of $x$ and $y$, respectively.

A topological space, which satisfies axiom $T_{i}$, is called a $T_{i}$-space. Each axiom $T_{i+1}$ is stronger than axiom $T_{i}$. Traditionally, $T_{2}$-spaces are called Hausdorff spaces (Kelley [24]).

Theorem 3.18. The topology $\tau_{c \mathrm{E}}$ in the space $\mathbb{R}^{\omega}$ does not satisfy the axiom $\left(T_{0}\right)$.

Proof. To prove the theorem, it is sufficient to take elements $\mathbf{0}=\left(a_{i}=0\right)_{i \in \omega}$ and $b=\left(b_{i}=\right.$ $\left.e^{-i}\right)_{i \in \omega}$. Any E-conical neighborhood of one of them includes the second point. Indeed, by the definition of an E-conical neighborhood of 0 , if $\mathrm{O} \mathbf{0}$ is an $\mathbf{E}$-conical neighborhood of $\mathbf{0}$, 
then there is $n \in \omega$ such that for all $i>n,\left(e^{-i} \in C_{i 1}\right)$. In this case, the sequence $c=\left(c_{i}\right)_{i \in \omega}$, in which $c_{i}=0$ for $i=1,2, \ldots, n$ and $b_{i}=e^{-i}$ for $i>n$, belongs to $O \mathbf{0}$. As the sequence $b=\left(b_{i}=e^{-i}\right)_{i \in \omega}$ is similar to the sequence $c$, it also belongs to $O \mathbf{0}$.

The proof for the neighborhoods of $b$ is similar.

Theorem 3.18 is proved.

Proposition 3.19. $\mathbb{R}^{\omega}$ is a topological abelian group (McCarty [32]) with respect to addition in $\mathbb{R}^{\omega}$ and topology $\tau_{c \mathrm{E}}$.

Remark 3.20. However, $\mathbb{R}^{\omega}$ is not a topological vector space with respect to $\tau_{c \mathrm{E}}$ although (Burgin [6]) $\mathbb{R}^{\omega}$ is a topological vector space with respect to $\tau_{s p}$.

To show this, let us consider number 1 with its neighborhood $O_{k} 1=\{a \in \mathbb{R} ; \mid a-$ $1 \mid<k\}$, where $k \in \mathbb{R}^{++}$and the sequence $\mathbf{1}=\left(c_{i}=1\right)_{i \in \omega}$. Then to be a topological vector space with respect to $\tau_{c \mathbf{E}}$, the space $\mathbb{R}^{\omega}$ needs the following property: for any $\mathbf{E}$-conical neighbourhood $O 1$ of the element 1 , there are neighborhoods $O_{k} 1$ and $O_{1} 1$ such that $O_{k} 1 \cdot O_{1} \mathbf{1} \subseteq 01$. The product $O_{k} 1 \cdot O_{1} \mathbf{1}$ always contains the set $O_{k} 1 \cdot \mathbf{1}=O_{k} \mathbf{1}$, which is a spherical neighborhood of $\mathbf{1}$. However, there are $\mathbf{E}$-conical neighbourhoods of the element $\mathbf{1}$, for example, $O_{1 / x} \mathbf{0}=\left\{c=\left(c_{i}\right)_{i \in \omega} \in \mathbb{R}^{\omega} ; \exists n \in \omega\right.$ for all $\left.i>n\left(\left|c_{i}\right|<1 / i\right)\right\}$, such that they do not contain any spherical neighborhood of $\mathbf{1}$. Thus, $\mathbb{R}^{\omega}$ is not a topological vector space with respect to $\tau_{c \mathrm{E}}$.

Lemma 3.21. The points $a, b \in \mathbb{R}^{\omega}$ determine the same $\mathbf{E}$-hypernumber if and only if any E-conical neighborhood of a contains $b$.

Proof

Necessity. Let the sequences $a=\left(a_{i}\right)_{i \in \omega}$ and $b=\left(b_{i}\right)_{i \in \omega}$ from $\mathbb{R}^{\omega}$ determine the same E-hypernumber, that is, $\alpha=\operatorname{Hn}^{\mathrm{E}}\left(a_{i}\right)_{i \in \omega}=\operatorname{Hn}^{\mathrm{E}}\left(b_{i}\right)_{i \in \omega}$, and let $O a$ be an E-conical neighborhood of $a$. Then there is $k \in \mathbb{R}^{++}$such that there is $m \in \omega$ such that for all $i>m$, we have $\left|a_{i}-b_{i}\right|<e^{-k i}$. Consequently, we have $a_{i}+\left|a_{i}-b_{i}\right|<a_{i}+e^{-k i}$ for any $i>m$. If $O a$ is an E-conical neighborhood of a sequence $a \in \mathbb{R}^{\omega}$, then $a \pm \mathbf{e}_{k}=\left(a_{i}\right)_{i \in \omega} \pm\left(e^{-k i}\right)_{i \in \omega}=$ $\left(a_{i} \pm e^{-k i}\right)_{i \in \omega} \in O a$. In addition, $\left|a_{i}-b_{i}\right|<e^{-k i}=\left|a_{i}-\left(a_{i}+e^{-k i}\right)\right|$. Consequently, by condition (3) for E-conical neighborhoods, the sequence $b=\left(b_{i}\right)_{i \in \omega}$ belongs to Oa.

Necessity is proved.

Sufficiency. Let us consider two sequences $a=\left(a_{i}\right)_{i \in \omega}$ and $b=\left(b_{i}\right)_{i \in \omega}$ from $\mathbb{R}^{\omega}$ that define different hypernumbers, that is, $\alpha=\operatorname{Hn}^{\mathrm{E}}\left(a_{i}\right)_{i \in \omega} \neq \beta=\operatorname{Hn}^{\mathrm{E}}\left(b_{i}\right)_{i \in \omega}$. Then the following statement is true:

$$
\forall k \in \mathbb{R}^{++}, \forall n \in \omega, \quad \exists i \geq n\left(\left|a_{i}-b_{i}\right|>e^{-k i}\right) .
$$

We define $n(1)$ equal to a number $n$ such that for some $i \geq n,\left(\left|a_{i}-b_{i}\right|>e^{-i}\right)$. Then $n(2)$ is equal to a number $n$ such that $n>n(1)$ and for some $i \geq n,\left(\left|a_{i}-b_{i}\right|>e^{-i / 2}\right)$. We continue this process, defining numbers $n(r)$, where $r \in \omega$ and $n(r)$ is equal to a number $n$ such that $n>n(r-1)$ and for some $i \geq n,\left(\left|a_{i}-b_{i}\right|>e^{-i / r}\right)$. It is possible to do this for any $r$ as the formula (3.1) is true for any number $n$. 
This allows us to define sets $C_{i}=\left(a_{i}-\left|a_{n(r)}-b_{n(r)}\right|, a_{i}+\left|a_{n(r)}-b_{n(r)}\right|\right)$ with $n(r) \leq$ $i<n(r+1)$ for all $r=1,2,3, \ldots$. Then we put $O a=\mathrm{Cl}_{\text {sim }}\left(\bigcup_{i=1}^{\infty} C_{i}\right)$ and show that it is an E-conical neighborhood of $a$, validating conditions (1)-(5) from Definition 3.3.

Condition 1. Let $c=\left(c_{i}\right)_{i \in \omega} \in O a$. Then there is $m \in \omega$ such that for all $i>m$ with $n(r) \leq$ $i<n(r+1)$, we have $\left|a_{i}-c_{i}\right|<\left|a_{i}-\left(a_{i}+\left|a_{n(r)}-b_{n(r)}\right|\right)\right|=\left|a_{n(r)}-b_{n(r)}\right|$ for all $r$ with $n(r)>m$. This implies that there is $m \in \omega$ such that for all $i>m$ with $n(r) \leq i<n(r+1)$, we have $\left|a_{i}-\left(a_{i}+\left|a_{i}-c_{i}\right|\right)\right|<\left|a_{i}-c_{i}\right|<\left|a_{i}-\left(a_{i}+\left|a_{n(r)}-b_{n(r)}\right|\right)\right|=\left|a_{n(r)}-b_{n(r)}\right|$ for all $r$ with $n(r)>m$. Consequently, $a+|c-a| \in O a$. In a similar way, we show that $a-|c-a| \in$ Oa.

Conditions 2-4. Conditions 2-4 for $\mathrm{Oa}$ follow directly from the definition of $\mathrm{Oa}$.

Condition 5. Given $e^{-t i}$, we can find a number $r \in \omega$ such that $t>r^{-1}$. Then $e^{-t i}<$ $e^{-i / r}$ for any $i \in \omega$. This implies $a_{n(r)}+e^{-t n(r)}<a_{n(r)}+e^{-n(r) / r}<a_{n(r)}+\left|a_{n(r)}-b_{n(r)}\right|$ and $a_{n(r)}-e^{-t n(r)}>a_{n(r)}-e^{-n(r) / r}>a_{n(r)}-\left|a_{n(r)}-b_{n(r)}\right|$. Consequently, $a_{n(r)}+e^{-t n(r)} \in C_{n(r)}$ and $a_{n(r)}-e^{-t n(r)} \in C_{n(r)}$. When $n(r) \leq i<n(r+1)$, we have $a_{i}+e^{-t i}<a_{i}+e^{-i / r}<a_{i}+$ $e^{-n(r) / r}<a_{i}+\left|a_{n(r)}-b_{n(r)}\right|$ and $a_{i}-e^{-t i}>a_{i}-e^{-i / r}>a_{i}-e^{-n(r) / r}>a_{i}-\left|a_{n(r)}-b_{n(r)}\right|$. Consequently, $a_{i}+e^{-t i} \in C_{i}$ and $a_{i}-e^{-t i} \in C_{i}$. When $n(r+1) \leq i<n(r+2)$, we have $a_{i}+$ $e^{-t i}<a_{i}+e^{-i / r}<a_{i}+e^{-i /(r+1)}<a_{i}+e^{-n(r+1) /(r+1)}<a_{i}+\left|a_{n(r+1)}-b_{n(r+1)}\right|$ and $a_{i}-e^{-t i}>$ $a_{i}-e^{-i / r}>a_{i}-e^{-i /(r+1)}>a_{i}-e^{-n(r+1) /(r+1)}>a_{i}-\left|a_{n(r+1)}-b_{n(r+1)}\right|$. Hence, $a_{i}+e^{-t i} \in C_{i}$ and $a_{i}-e^{-t i} \in C_{i}$. We continue this process, demonstrating by induction that condition (5) is true for $O a$.

Let $k$ be an arbitrary natural number and $n(r+k) \leq i<n(r+k+1)$. Then $a_{i}+e^{-t i}<$ $a_{i}+e^{-i / r}<a_{i}+e^{-i /(r+k)}<a_{i}+e^{-n(r+k) /(r+k)}<a_{i}+\left|a_{n(r+k)}-b_{n(r+k)}\right|$ and, in a similar way, $a_{i}-e^{-t i}>a_{i}-e^{-i / r}>a_{i}-e^{-i /(r+k)}>a_{i}-e^{-n(r+k) /(r+k)}>a_{i}-\left|a_{n(r+k)}-b_{n(r+k)}\right|$. Consequently, $a_{i}+e^{-t i} \in C_{i}$ and $a_{i}-e^{-t i} \in C_{i}$. As $k$ is an arbitrary natural number, this implies that $a \pm \mathbf{e}_{t}=\left(a_{i}\right)_{i \in \omega} \pm\left(e^{-t i}\right)_{i \in \omega}=\left(a_{i} \pm e^{-t i}\right)_{i \in \omega} \in O a$.

Thus, $\mathrm{Oa}$ is an E-conical neighborhood of $a$. By Lemma 3.17, (1/2)॰Oa is also an E-conical neighborhood of the sequence $a$. At the same time, $b \notin(1 / 2) \circ O a$.

By the principle of excluded middle, sufficiency is also proved. This concludes the proof of the Lemma.

Lemma 3.21 implies that the topology $\tau_{c \mathrm{E}}$ is stable with respect to E-hypernumbers, that is, if points $a=\left(a_{i}\right)_{i \in \omega}, b=\left(b_{i}\right)_{i \in \omega} \in \mathbb{R}^{\omega}$ determine the same E-hypernumber, then any $\mathbf{E}$-conical neighborhood of $a$ contains $b$, and vice versa.

As $\mathbf{E} \mathbb{R}_{\omega}$ is the quotient space of $\mathbb{R}^{\omega}$, the topology $\tau_{c \mathbf{E}}$ induces in $\mathbf{E} \mathbb{R}_{\omega}$ the definite topology $\delta_{c \mathrm{E}}$, which is generated by means of the projections of the E-conical neighborhoods, that is, if $p_{\mathbf{E}}: \mathbb{R}^{\omega} \rightarrow \mathbf{E} \mathbb{R}_{\omega}$ is the natural projection and $u \in \mathbf{E} \mathbb{R}_{\omega}$, then any neighborhood of $u$ is the image of some E-conical neighborhood of a point $a \in \mathbb{R}^{\omega}$ such that $p(a)=u$.

Remark 3.22. In contrast to topology $\delta_{s p}$ induced by $\tau_{s p}$ in $\mathbf{E} \mathbb{R}_{\omega}$, topology $\delta_{c \mathbf{E}}$ in $\mathbf{E} \mathbb{R}_{\omega}$ does not induce the natural topology for real numbers $\mathbb{R}$ as a subset of $\mathbb{R}^{\omega}$. In topology $\delta_{c \mathrm{E}}$, the natural inclusion of $\mathbb{R}$ in $\mathbf{E} \mathbb{R}_{\omega}$ has discrete topology.

Indeed, topology $\delta_{c \mathbf{E}}$ in $\mathbf{E R}_{\omega}$ is induced by topology $\tau_{c \mathrm{E}}$, while $\mathbb{R}$ is a discrete subset of $\mathbb{R}^{\omega}$ in topology $\tau_{c \mathrm{E}}($ Remark 3.11).

Proposition 3.9 implies the following result. 
Corollary 3.23. Topology $\delta_{c \mathbf{E}}$ in $\mathbb{R}^{\omega}$ is stronger than topology $\delta_{s p}$.

Properties of the induced topology $\delta_{c \mathrm{E}}$ are better than similar properties of the topology $\tau_{c \mathrm{E}}$.

Theorem 3.24. The topology $\delta_{c \mathrm{E}}$ satisfies axiom $\left(T_{2}\right)$, and thus $\mathbf{E} \mathbb{R}_{\omega}$ is a Hausdorff space.

Proof. Let us consider two arbitrary hypernumbers $\alpha$ and $\beta$ from the set $\mathbf{E R}_{\omega}$. If $\alpha \neq$ $\beta$ in $\mathbf{E R}_{\omega}$, then any sequences $a=\left(a_{i}\right)_{i \in \omega} \in \alpha$ and $b=\left(b_{i}\right)_{i \in \omega} \in \beta$ satisfy the following condition (cf. Section 2): for any positive number $k$ and any $n \in \omega$ there is an $i>n$ such that $\left|a_{i}-b_{i}\right|>e^{-k j}$. It makes possible to choose for a given positive number $k$ an infinite set $M$ of natural numbers such that for any $m \in M$ the inequality $\left|a_{m}-b_{m}\right|>e^{-k j}$ is valid.

Let us take the E-conical neighborhood $\mathrm{Oa}$ of a constructed in the proof of Lemma 3.21. By the definition $O a=\mathrm{Cl}_{\text {sim }}\left(\bigcup_{i=1}^{\infty} C_{i}\right)$, where $C_{i}=\left(a_{i}-\left|a_{n(r)}-b_{n(r)}\right|, a_{i}+\mid a_{n(r)}-\right.$ $\left.b_{n(r)} \mid\right)$ with $n(r) \leq i<n(r+1)$ for all $r=1,2,3, \ldots$.

In a similar way, we construct the E-conical neighborhood $\mathrm{O} b$ of $b$ defined as $\mathrm{Ob}=$ $\mathrm{Cl}_{\text {sim }}\left(\cup_{i=1}^{\infty} \mathbf{D}_{i}\right)$, where $\mathbf{D}_{i}=\left(b_{i}-\left|a_{n(r)}-b_{n(r)}\right|, b_{i}+\left|a_{n(r)}-b_{n(r)}\right|\right)$ with $n(r) \leq i<n(r+1)$ for all $r=1,2,3, \ldots$.

By Lemma 3.14, $\mathrm{O}_{4} a=(1 / 4) \cdot \mathrm{O} a$ and $\mathrm{O}_{4} b=(1 / 4) \cdot \mathrm{O} b$ are also E-conical neighborhoods of the points $a$ and $b$, correspondingly. The projections $p\left(O_{4} a\right)$ and $p\left(O_{4} b\right)$ of these neighborhoods will be E-conical neighborhoods of $\alpha$ and $\beta$ with respect to the topology $\delta_{c \mathrm{E}}$. Moreover, $p\left(\mathrm{O}_{4} a\right) \cap p\left(\mathrm{O}_{4} b\right)=\varnothing$. To prove this, we suppose that there is $\gamma \in \mathbb{R}_{\omega}$ and $\gamma$ is an element of the set $p\left(O_{4} a\right) \cap p\left(O_{4} b\right)$. It implies that there are such points $u, v \in \mathbb{R}^{\omega}$, for which $p(u)=p(v)=\gamma, p: \mathbb{R}^{\omega} \rightarrow \mathbb{R}_{\omega}$ is a natural projection, and $u \in O_{4} a, v \in O_{4} b$.

Let $u=\left(u_{i}\right)_{i \in \omega}$ and $v=\left(v_{i}\right)_{i \in \omega}$. The equality $p(u)=p(v)$, Lemma 3.21, and the definition of E-hypernumbers imply that there is a number $k>0$ for which the following condition is valid: there is $m \in \omega$ such that for all $i>m\left(\left|u_{i}-v_{i}\right|<(1 / 3) e^{-k i}\right)$. At the same time, the set $M$, which is determined above, is infinite. So, there is a number $j \in M$, which is greater than $m$. For this $j$, we have $\left|u_{j}-v_{j}\right| \geq\left|a_{j}-b_{j}\right|-\left|a_{j}-u_{j}\right|-$ $\left|b_{j}-v_{j}\right| \geq e^{-k j}-(1 / 4) e^{-k j}-(1 / 4) e^{-k j}=(1 / 2) e^{-k j}>(1 / 3) e^{-k j}$. It contradicts the condition $\left|u_{j}-v_{j}\right|<(1 / 3) e^{-k j}$.

Consequently, the assumption is not true, and $p\left(O_{4} a\right) \cap p\left(O_{4} b\right)=\varnothing$. Theorem 3.24 is proved because $\alpha$ and $\beta$ are arbitrary points from $\mathbf{E R}_{\omega}$ and both $p\left(O_{4} a\right)$ and $p\left(O_{4} b\right)$ are E-conical neighborhoods of $\alpha$ and $\beta$, respectively.

This result makes it possible to give an axiomatic description of $\mathbb{R}_{\omega}$.

TheOREM 3.25. The space $\mathrm{ER}_{\omega}$ with the topology $\delta_{c \mathrm{E}}$ is the largest Hausdorff quotient space of the topological space $\mathbb{R}^{\omega}$ with the topology $\tau_{c \mathrm{E}}$.

In other words, if a Hausdorff space $X$ is a quotient space of $\mathbb{R}^{\omega}$ with the continuous projection $q: \mathbb{R}^{\omega} \rightarrow X$, then there is a continuous projection $v: \mathbb{E}_{\omega} \rightarrow X$ for which $q=$ $p v$, that is, the following diagram is commutative:

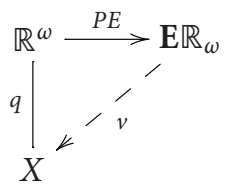


Proof. By Theorem 3.24, ER $\mathbb{R}_{\omega}$ is a Hausdorff space. Thus, to prove the theorem, it is necessary to demonstrate that if a Hausdorff space $X$ is a quotient space of $\mathbb{R}^{\omega}$ with the continuous projection $q: \mathbb{R}^{\omega} \rightarrow X$, then there is a continuous projection $v: \mathbb{E}_{\omega} \rightarrow X$ for which $q=p_{\mathrm{E}} v$.

Let us consider such a Hausdorff space $X$ with the continuous projection $q: \mathbb{R}^{\omega} \rightarrow X$. Then, for any points $x, y \in X$, the inequality $x \neq y$ implies existence of neighborhoods $O x$ and $O y$ for which $O x \cap O y=\varnothing$ is valid. As $X$ is a quotient space of $\mathbb{R}^{\omega}$, there are points $a, b \in \mathbb{R}^{\omega}$ for which $q(a)=x$ and $q(b)=y$. The inverse images $q^{-1}(O x)$ and $q^{-1}(O y)$ are open sets because $q$ is a continuous mapping. Besides, $a \in q^{-1}(O x)$ and $b \in q^{-1}(O y)$ because $q(a)=x$ and $q(b)=y$. That is why $q^{-1}(O x)$ contains an E-conical neighborhood $\mathrm{O} a$ of $a$, and $q^{-1}(\mathrm{O} y)$ contains an E-conical neighborhood $\mathrm{O} b$ of $b$.

Let us suppose that $p_{\mathrm{E}}(a)=p_{\mathrm{E}}(b)$. Then by Lemma 3.21, $a \in O b$ and $b \in O a$. As a consequence, $x=q(a) \in q(O b) \subseteq O y$ and $y=q(b) \in q(O a) \subseteq O x$. It contradicts the condition that $O x \cap O y=\varnothing$. Thus, for arbitrary points $x$ and $y$ from $X$ and elements $a$ and $b$ from $\mathbb{R}^{\omega}$ such that $q(a)=x$ and $q(b)=y$, we have $p_{\mathrm{E}}(a) \neq p_{\mathrm{E}}(b)$.

This makes it possible to define the mapping $v: \mathbb{E R}_{\omega} \rightarrow X$ as follows: $v(x)=p(a)$ for any $x \in X$ and for $a \in \mathbb{R}^{\omega}$ such that $q(a)=x$. The definition of $v$ implies that the mapping $v$ is continuous because $p_{\mathrm{E}} v=q$ and the topology $\delta_{c \mathrm{E}}$ in $\mathbf{E R}_{\omega}$ is induced by the topology $\tau_{c \mathrm{E}}$ in $\mathbb{R}^{\omega}$, that is, $p_{\mathrm{E}}$ is an open mapping.

Theorem 3.25 is proved.

Theorem 3.25 gives an axiomatic topological characterization of the space $\mathbf{E R}_{\omega}$ of real E-hypernumbers as the largest Hausdorff quotient space of the topological space $\mathbb{R}^{\omega}$ with the topology $\tau_{c \mathrm{E}}$. The condition that the space $R^{\omega}$ has the topology $\tau_{c \mathrm{E}}$ is essential because in the discrete topology, for example, there are larger quotient spaces of $\mathbb{R}^{\omega}$.

Theorem 3.26. The set $\mathbf{E}^{\mathbf{D}} \mathbb{R}_{\omega}$ of all $\mathbf{D}$-moderate real $\mathbf{E}$-hypernumbers is an open subspace of the topological space $\mathbf{E R}_{\omega}$.

Proof. Let us consider a D-moderate sequence $a=\left(a_{i}\right)_{i \in \omega} \in \mathbb{R}^{\omega}$ and the $\mathbf{D}$-moderate real E-hypernumber $\alpha=\operatorname{Hn}\left(a_{i}\right)_{i \in \omega}$. By the definition of $\mathbf{D}$-moderate sequences, we have $a_{i}<$ $i^{n}$ starting from some $i=m$. The set $\mathbf{O 0}=\left\{c=\left(c_{i}\right)_{i \in \omega} \in \mathbb{R}^{\omega} ;|c|<\mathbf{i}^{\mathbf{n}+1}\right\}$ is an E-conical neighborhood of $\mathbf{0}=\left(c_{i}=0\right)_{i \in \omega}$. The proof is the same as given in Example 3.6 for the case $n=1$.

Consequently, the set $\mathrm{Oa}=a+O \mathbf{O}$ is an E-conical neighborhood of $a$. As all elements from $O 0$ belong to $\mathbf{D} \mathbb{R}^{\omega}$, all elements from $O a$ belong to $\mathbf{D} \mathbb{R}^{\omega}$. As $a$ is an arbitrary element from $\mathbf{D} \mathbb{R}^{\omega}$, the set $\mathbf{D} \mathbb{R}^{\omega}$ is open in $\mathbb{R}^{\omega}$. As topology in $\mathbf{E} \mathbb{R}_{\omega}$ is determined by the projection $p_{\mathbf{E}}: \mathbb{R}^{\omega} \rightarrow \mathbb{E R}_{\omega}$ and $\mathbf{E}^{\mathbf{D}} \mathbb{R}_{\omega}$ is the image of an open set $\mathbf{D} \mathbb{R}^{\omega}$, the set $\mathbf{E}^{\mathbf{D}} \mathbb{R}_{\omega}$ is also open.

Theorem 3.26 is proved.

We study how topology in spaces $\mathbf{E} \mathbb{R}_{\omega}$ and $\mathbf{E}^{\mathbf{D}} \mathbb{R}_{\omega}$ is correlated with algebraic structures in these sets. To do this, we need some general results for topological, universal, and heterogeneous algebras. We recall basic concepts from the theory of topological, linear, universal, and heterogeneous algebras (Cohn [12]; Kurosh [27]; Burgin [5]; Mallios [30]).

Definition 3.27. A heterogeneous or multibase universal algebra $\mathbf{A}$ is a set $A$ with a system of operations $\Sigma$ in which elements of $A$ form an indexed system $A=\left\{A_{i}\right\}$ of sets and each operation is a mapping having the form $\omega: A_{i 1} \times A_{i 2} \times \cdots \times A_{i k} \rightarrow A_{i}$. 
A universal algebra is a heterogeneous algebra in which $A$ consists of a single set.

Definition 3.28. The system $A$ is called the carrier of the heterogeneous algebra $\mathbf{A}$.

Examples of heterogeneous universal algebras are vector spaces, linear algebras, modules, polygons (i.e., sets on which monoids act), automata or state machines, polyadic or Halmos algebras (Halmos [18]), and nonhomogeneous polyadic algebras (LeBlanc [28]). Heterogeneous universal algebras were studied by several authors under different names. To mention only some of them, it is necessary to name algebras with a scheme of operators introduced by Higgins $[19,20]$, heterogeneous algebras from the papers of Birkhoff and Lipson [2] and Matthiessen [31], and many-sorted algebras studied by Plotkin [38]. The term "heterogeneous algebras" is used more often than other related terms. Heterogeneous (multibase or many-sorted) algebras represent the next level of the development of algebra. Namely, in ordinary (or homogeneous) universal algebras operations are defined on a set, while in heterogeneous algebras operations are defined on a named set (Burgin [10]). This makes it possible to develop more adequate models for many processes.

Let $\mathbf{A}=\left(A=\left\{A_{i}\right\}, \boldsymbol{\Sigma}\right)$ and $\mathbf{B}=\left(B=\left\{B_{i}\right\}, \boldsymbol{\Sigma}\right)$ be heterogeneous algebras.

Definition 3.29. A system of mappings $f=\left\{f_{i}: A_{i} \rightarrow A_{j}\right\}$ defined for all $i$ is called a homomorphism of a heterogeneous algebra $\mathbf{A}$ into a heterogeneous algebra $\mathbf{B}$ if for any operation $\omega$ from $\Sigma$ the following identity is true: $f\left(\omega\left(x_{1}, x_{2}, \ldots, x_{k}\right)\right)=\omega\left(f\left(x_{1}\right), f\left(x_{2}\right), \ldots\right.$, $\left.f\left(x_{k}\right)\right)$.

Definition 3.30. The homomorphism $f: \mathbf{A} \rightarrow \mathbf{B}$ is called an epimorphism if all $f_{i}$ are projections.

Definition 3.31. A heterogeneous algebra $\mathbf{A}$ is topological if all sets $A_{i}$ are topological spaces and each operation is a continuous mapping of the form $\omega: A_{i 1} \times A_{i 2} \times \cdots \times$ $A_{i k} \rightarrow A_{i}$.

Theorem 3.32. If $\mathbf{A}$ is a heterogeneous topological algebra, $\mathbf{B}$ is a heterogeneous algebra, $p: \mathbf{A} \rightarrow \mathbf{B}$ is an epimorphism, $\mathbf{B}$ has topology induced by topology in $\mathbf{A}$, then $\mathbf{B}$ is also a heterogeneous topological algebra.

Proof. We need to prove that taking an element $u=\omega\left(u_{1}, u_{2}, \ldots, u_{k}\right)$ from the algebra B and its neighborhood $\mathrm{Ou}$, we can find neighborhoods $\mathrm{O} u_{1}, \mathrm{Ou}_{2}, \ldots, O u_{k}$ such that $\omega\left(O u_{1}, O u_{2}, \ldots, O u_{k}\right) \subseteq O u$. As $p: \mathbf{A} \rightarrow \mathbf{B}$ is a projection, there are elements $a_{1}, a_{2}, \ldots, a_{k}$ from the algebra $\mathbf{A}$ such that $u_{i}=p\left(a_{i}\right)$ for all $i=1,2, \ldots, k$. As $p: \mathbf{A} \rightarrow \mathbf{B}$ is an epimorphism, $u=\omega\left(u_{1}, u_{2}, \ldots, u_{k}\right)=\omega\left(p\left(a_{1}\right), p\left(a_{2}\right), \ldots, p\left(a_{k}\right)\right)=p\left(\omega\left(a_{1}, a_{2}, \ldots, a_{k}\right)\right)$. As topology in $\mathbf{B}$ is induced by the topology in $\mathbf{A}$, there is a neighborhood $\mathrm{Oa}$ of the element $a=$ $\omega\left(a_{1}, a_{2}, \ldots, a_{k}\right)$ such that $p(O a)=O u$. As $\mathbf{A}$ is a heterogeneous topological algebra, there are neighborhoods $O a_{1}, O a_{2}, \ldots, O a_{k}$ such that $\omega\left(O a_{1}, O a_{2}, \ldots, O a_{k}\right) \subseteq O a$. As topology in $\mathbf{B}$ is induced by the topology in $\mathbf{A}$, the projections $p\left(O a_{1}\right), p\left(O a_{2}\right), \ldots, p\left(O a_{k}\right)$ are neighborhoods of the elements $u_{1}, u_{2}, \ldots, u_{k}$ such that $\omega\left(p\left(O a_{1}\right), p\left(O a_{2}\right), \ldots, p\left(O a_{k}\right)\right)=$ $p\left(\omega\left(O a_{1}, O a_{2}, \ldots, O a_{k}\right)\right) \subseteq p(O a)=O u$.

Theorem 3.32 is proved.

Theorem 3.32 and Proposition 3.9 imply the following result. 
Corollary 3.33. The set $\mathbf{E}_{\omega}$ with the topology $\delta_{c \mathbf{E}}$ is a topological abelian group.

For topology $\tau_{s p}$, we have a stronger result.

Theorem 3.34 (Burgin [4]). The set $\mathbb{R}^{\omega}$ with the topology $\tau_{s p}$ is a topological vector space over the field $\mathbb{R}$ with the natural topology, and the set $\mathbf{D} \mathbb{R}^{\omega}$ of all $\mathbf{D}$-moderate sequences is its topological subspace.

Proof. The topology $\tau_{s p}$ in $\mathbb{R}^{\omega}$ is defined by spherical neighborhoods. A spherical neighborhood of a sequence $a=\left(a_{i}\right)_{i \in \omega} \in \mathbb{R}^{\omega}$ is an arbitrary set $O_{k} a=\left\{c=\left(c_{i}\right)_{i \in \omega} \in \mathbb{R}^{\omega} ; \exists n \in \omega\right.$ for all $\left.i>n\left(\left|a_{i}-c_{i}\right|<k\right)\right\}$ with $k \in \mathbb{R}^{++}$. To prove that $\mathbb{R}^{\omega}$ with the topology $\tau_{s p}$ is a topological linear algebra, we need to show that operations of addition $a+b$, multiplication $a \cdot b$, and multiplication by a real number $r \cdot a$ are continuous mappings (Mallios [30]).

Let us consider a spherical neighborhood $O_{k}(a+b)$ of the sum $a+b$. Taking $h=1 / 2 k$, we have $O_{h} a+O_{h} b \subseteq O_{k}(a+b)$. So, addition is a continuous mapping.

It follows from the definitions that the product $O_{h}(r) \cdot O_{k}(a)$ is a spherical neighborhood $O_{h k}(r \cdot a)$ of the product $r \cdot a$. Thus, $\mathbb{R}^{\omega}$ with the topology $\tau_{s p}$ is a topological linear algebra.

By the definition (cf. Section 2), the set $\mathbf{D} \mathbb{R}^{\omega}$ is a subspace of $\mathbb{R}^{\omega}$. At the same time, the set $\mathbf{D} \mathbb{R}^{\omega}$ is an open subset of $\mathbb{R}^{\omega}$. Consequently, $\mathbf{D} \mathbb{R}^{\omega}$ is a topological subspace of $\mathbb{R}^{\omega}$.

Theorem 3.34 is proved.

Remark 3.35. However, $\mathbb{R}^{\omega}$ with the topology $\tau_{s p}$ is not a topological linear algebra over the field $\mathbb{R}$ with the natural topology.

Indeed, if we consider sequences $\mathbf{0}=\left(c_{i}=0\right)_{i \in \omega}$ and $\mathbf{i}_{+}=\left(c_{i}=i\right)_{i \in \omega}$, then $\mathbf{0} \cdot \mathbf{i}_{+}=\mathbf{0}$. However, no spherical neighborhood of $\mathbf{0}$ contains the product $\mathrm{O}_{k} \mathbf{0} \cdot \mathrm{O}_{h} \mathbf{i}_{+}$of neighborhoods $O_{k} \mathbf{0}$ and $O_{h} \mathbf{i}_{+}$whatever small numbers $k$ and $h$ we would take.

While $\mathbf{E}^{\mathbf{D}} \mathbb{R}_{\omega}$ with the topology $\delta_{s p}$ is a topological vector space over the field $\mathbb{R}$ with the natural topology, $\mathbf{E}^{\mathbf{D}} \mathbb{R}_{\omega}$ with the topology $\delta_{c \mathbf{E}}$ is not a topological vector space over the field $\mathbb{R}$ with the natural topology. However, if we take the field $\mathbb{R}$ with the discrete topology, we have the following result.

THeOREM 3.36. The set $\mathbb{R}^{\omega}$ with the topology $\tau_{c \mathrm{E}}$ is a topological vector space over the field $\mathbb{R}$ with the discrete topology.

Proof. To prove that $\mathbb{R}^{\omega}$ with the topology $\tau_{c \mathrm{E}}$ is a topological vector space, we need to show that operations of addition $a+b$ and multiplication by a real number $r \cdot a$ are continuous mappings.

By the definition of conical neighborhoods, for an arbitrary conical neighborhood $O(a+b)$ of the sum $a+b$, we have $O(a+b)=O 0+(a+b)$ for some conical neighborhood $O \mathbf{O}(a+b)$ of $\mathbf{0}$. Then by Lemma 3.14, Oa =1/2O0+a is a conical neighborhood of $a$, $\mathrm{O} b=1 / 2 \mathrm{O} 0+b$ is a conical neighborhood of $b$, and $\mathrm{O} a+\mathrm{O} b=1 / 2 \mathrm{O} 0+a+1 / 2 \mathrm{O} 0+b \subseteq$ $\mathrm{O} 0+(a+b)=O(a+b)$. So, addition is a continuous mapping.

Multiplication by a real number $r \cdot a$ is a continuous mapping because any $r$ is an open set in the discrete topology and, by Lemma 3.14, the $r \cdot \mathrm{O} a$ is an E-conical neighborhood of the sequence $r \cdot a \in \mathbb{R}^{\omega}$ for any E-conical neighborhood $O a$ of the sequence $a$.

Theorem 3.36 is proved. 
As it is proved in Burgin [7], $\mathbb{R}_{\omega}$ is a vector space over $\mathbb{R}$ and, by Theorem 2.50, $\mathbb{R}_{\omega}$ is a vector space over $\mathbb{R}$. Thus, for the topology $\delta_{s p}$ induced by the topology in $\mathbb{R}^{\omega}$, Theorems 3.32 and 3.34 imply the following result.

Theorem 3.37. The sets $\mathbb{R}_{\omega}$ with the topology induced by the topology in $\mathbb{R}^{\omega}$ and $\mathbb{E}_{\omega}$ with the topology $\delta_{s p}$ are topological vector spaces over the field $\mathbb{R}$ with the natural topology.

As it is proved in Section 2, $\mathbf{E}^{\mathbf{D}} \mathbb{R}_{\omega}$ is a subspace of $\mathbf{E R}_{\omega}$. Thus, with the topology $\delta_{s p}$ induced by the topology in $\mathbb{R}^{\omega}$, Theorems 3.32 and 3.34 imply the following result.

Theorem 3.38. The set $\mathbf{E}^{\mathrm{D}} \mathbb{R}_{\omega}$ with the topology $\delta_{s p}$ is a topological vector space over the field $\mathbb{R}$ with the natural topology.

However, similar to $\mathbb{R}^{\omega}$, the set $\mathbf{E}^{\mathbf{D}} \mathbb{R}_{\omega}$ with the topology $\delta_{s p}$ is not a topological linear algebra over the field $\mathbb{R}$ with the natural topology.

While $\mathbf{E} \mathbb{R}_{\omega}$ with the topology $\delta_{s p}$ is a topological vector space, $\mathbf{E} \mathbb{R}_{\omega}$ with the topology $\delta_{c \mathrm{E}}$ is not a topological vector space over the field $\mathbb{R}$ with the natural topology. However, Theorems 3.32 and 3.36 imply the following result.

Theorem 3.39. The set $\mathbf{E R}_{\omega}$ with the topology $\delta_{c \mathrm{E}}$ is a topological vector space over the field $\mathbb{R}$ with the discrete topology.

At the same time, $\mathbf{E}^{\mathrm{D}} \mathbb{R}_{\omega}$ with the topology $\delta_{c \mathbf{E}}$ is also not a topological linear algebra and even not a topological vector space over the field $\mathbb{R}$ with the natural topology. However, if we take the field $\mathbb{R}$ with the discrete topology, Theorems 3.32 and 3.36 allow us to prove that $\mathbf{E}^{\mathrm{D}} \mathbb{R}_{\omega}$ with the topology $\delta_{c \mathbf{E}}$ is a topological linear algebra. Before doing this, we need the following result.

Definition 3.40. If $\mathbf{i}^{\mathbf{n}}=\left(1 / i^{n}\right)_{i \in \omega}$, then $\mathbf{i}^{\mathbf{n}} \cdot O a=\mathrm{Cl}_{\operatorname{sim}}\left(\left\{d=\left(d_{i}\right)_{i \in \omega} ; d=\mathbf{i}^{\mathbf{n}} \cdot c=\left(\left(1 / i^{n}\right)\right.\right.\right.$ $\left.\left.\left.c_{i}\right)_{i \in \omega} ; c=\left(c_{i}\right)_{i \in \omega} \in O a\right\}\right)$.

LEMma 3.41. If $\mathrm{O} \mathbf{0}$ is an $\mathbf{E}$-conical neighborhood of a sequence $\mathbf{0} \in \mathbb{R}^{\omega}$, then for any $n \in \mathbb{N}$, $\mathbf{i}^{\mathbf{n}} \cdot \mathrm{O} \mathbf{0}$ is an $\mathbf{E}$-conical neighborhood of the sequence $\mathbf{0 .}$

Proof. To show that $\mathbf{i}^{\mathbf{n}} \cdot \mathrm{O} \mathbf{0}$ is an $\mathbf{E}$-conical neighborhood of $\mathbf{0}$, we need to check conditions (1)-(5) from Definition 3.3.

Condition 1. Let $c=\left(c_{i}\right)_{i \in \omega} \in \mathbf{i}^{\mathbf{n}} \cdot$ O0. Then $c \approx \mathbf{i}^{\mathbf{n}} \cdot b$ for some $b=\left(b_{i}\right)_{i \in \omega} \in O \mathbf{0}$. By condition (1), we have $|b| \in O \mathbf{0}$ and $-|b| \in O \mathbf{0}$. Thus, $\left|\mathbf{i}^{\mathbf{n}} \cdot b\right|=\mathbf{i}^{\mathbf{n}} \cdot|b| \in \mathbf{i}^{\mathbf{n}} \cdot O \mathbf{0}$ and $-\left|\mathbf{i}^{\mathbf{n}} \cdot b\right|=-\mathbf{i}^{\mathbf{n}} \cdot|b|=\mathbf{i}^{\mathbf{n}} \cdot(-|b|) \in \mathbf{i}^{\mathbf{n}} \cdot O \mathbf{0}$. At the same time, $c \approx \mathbf{i}^{\mathbf{n}} \cdot b$ implies $|c| \approx$ $\left|\mathbf{i}^{n} \cdot b\right|$. As the set $\mathbf{i}^{\mathbf{n}} \cdot O \mathbf{O}$ is closed with respect to similar elements, we have $|c| \in \mathbf{i}^{\mathbf{n}} \cdot O \mathbf{0}$ and $-|c| \in \mathbf{i}^{\mathbf{n}} \cdot O \mathbf{0}$, that is, condition (1) is true for $\mathbf{i}^{\mathbf{n}} \cdot O \mathbf{0}$.

Condition 2. This condition is true for $\mathbf{i}^{\mathbf{n}} \cdot \mathrm{O} \mathbf{0}$ because similarity closure is an idempotent operation (Kuratowski [25, Axiom 4]).

Condition 3. Let $d=\left(d_{i}\right)_{i \in \omega} \in \mathbb{R}^{\omega}$, and for some $c=\left(c_{i}\right)_{i \in \omega}$ from $\mathbf{i}^{\mathbf{n}} \cdot \mathbf{O 0}$, the following condition is true: there is $m \in \omega$ such that for all $i>m\left(\left|d_{i}\right| \leq\left|c_{i}\right|\right)$. Then for the same $m \in$ $\omega$, we have $i^{n} \cdot\left|d_{i}\right| \leq i^{n} \cdot\left|c_{i}\right|$ when $i>m$. By condition (2) for O0, $\mathbf{i}^{-\mathbf{n}} \cdot|c| \in O \mathbf{0}$. Then 
by condition (3) for $O \mathbf{0}, \mathbf{i}^{-\mathbf{n}} \cdot|d| \in O \mathbf{0}$. Consequently, $|d|=\mathbf{i}^{\mathbf{n}} \cdot\left(\mathbf{i}^{-n} \cdot|d|\right) \in \mathbf{i}^{-\mathbf{n}} \cdot O \mathbf{0}$. Lemma 3.8 implies that $d \in O \mathbf{0}$, that is, condition (3) is true for $\mathbf{i}^{\mathbf{n}} \cdot \mathbf{O 0}$.

Condition 4. Let $d=\left(d_{i}\right)_{i \in \omega} \in \mathbf{i}^{\mathbf{n}} \cdot$ O0. Then $d \approx r \cdot b$ for some $b=\left(b_{i}\right)_{i \in \omega} \in O \mathbf{0}$. Then there is $a=\left(a_{i}\right)_{i \in \omega} \in O \mathbf{0}$ such that $b>a$ and there is $c=\left(c_{i}\right)_{i \in \omega} \in O \mathbf{0}$ such that $c>b$. As all $1 / i^{n} \in \mathbb{R}^{++}, \mathbf{i}^{\mathbf{n}} \cdot b>\mathbf{i}^{\mathbf{n}} \cdot a$ and $\mathbf{i}^{\mathbf{n}} \cdot c>\mathbf{i}^{\mathbf{n}} \cdot b$. Then $d>\mathbf{i}^{\mathbf{n}} \cdot a$ and $\mathbf{i}^{\mathbf{n}} \cdot c>d$. Besides, $\mathbf{i}^{\mathbf{n}} \cdot a$ and $\mathbf{i}^{\mathbf{n}} \cdot c$ belong to $\mathbf{i}^{\mathbf{n}} \cdot O \mathbf{0}$. It means that condition (4) is true for $\mathbf{i}^{\mathbf{n}} \cdot O \mathbf{0}$.

Condition 5. Let us consider an arbitrary function $e^{-h i}$ from $\mathbf{E}^{-1}$ and the sequence $\mathbf{e}^{-h i}=$ $\left(e^{-h i}\right)_{i \in \omega}$. Let us take some number $d \in \mathbb{R}^{++}$such that $h-d>0$. Then $\exists m \in \omega$ for all $i>$ $m\left(e^{d i}>1 / i^{n}\right)$. By the definition, $\mathbf{e}^{-(h-d) i}=\left(e^{-(h-d) i}\right)_{i \in \omega} \in O \mathbf{0}$. Then $\mathbf{e}^{-h i}<\mathbf{i}^{\mathbf{n}} \cdot \mathbf{e}^{-(h-d) i} \in$ $\mathbf{i}^{\mathbf{n}} \cdot O \mathbf{0}$ and, by condition (3), which has been already proved, $\mathbf{e}^{-h i} \in \mathbf{i}^{\mathbf{n}} \cdot O \mathbf{0}$, that is, condition (5) is true for $O \mathbf{0 .}$

The lemma is proved.

TheOREm 3.42. The set $\mathbf{E}^{\mathbf{D}} \mathbb{R}_{\omega}$ with topology $\delta_{c \mathrm{E}}$ is a topological linear algebra over the field $\mathbb{R}$ with the discrete topology.

Proof. The results of Theorems 3.32 and 3.36 show that we need only to prove that multiplication $a \cdot b$ in $\mathbf{E}^{\mathbf{D}} \mathbb{R}_{\omega}$ is a continuous mapping.

Let us take two elements $a$ and $b$ from $\mathbf{E}^{\mathbf{D}} \mathbb{R}_{\omega}$ and consider an E-conical neighborhood $O(a \cdot b)$ of the product $a \cdot b$. Then $O(a \cdot b)=O \mathbf{O}+a \cdot b$ for some E-conical neighborhood $O \mathbf{0}$ of $\mathbf{0}$. Let us take the set $O_{1} \mathbf{0}=O \mathbf{0} \cap O_{1 / 2} \mathbf{0}$, where $O_{1 / 2} \mathbf{0}=\left\{c=\left(c_{i}\right)_{i \in \omega} \in \mathbb{R}^{\omega}\right.$; there is $n \in \omega$ such that for all $\left.i>n,\left(\left|c_{i}\right|<1 / 2\right)\right\}$. The set $O_{1} \mathbf{0}$ is an $\mathbf{E}$-conical neighborhood of $\mathbf{0}$ because the intersection of the E-conical neighborhood $\mathrm{O} 0$ and spherical neighborhood $\mathrm{O}_{1 / 2} \mathbf{0}$ of $\mathbf{0}$ is an $\mathbf{E}$-conical neighborhood of $\mathbf{0}$, as any spherical neighborhood is an open set in the topology $\delta_{c \mathrm{E}}$ (cf. Proposition 3.10 and Corollary 3.23). By the definition, $O_{1} \mathbf{0}$. $O_{1} \mathbf{0} \subseteq(1 / 2) \cdot O_{1} \mathbf{0} \subseteq O_{1} \mathbf{0}$ because all element from $O_{1} \mathbf{0}$ have norms less than $1 / 2$. As both elements $a$ and $b$ belong to $\mathbf{E}^{\mathrm{D}} \mathbb{R}_{\omega}$, there are numbers $n$ and $m$ such that $a<\mathbf{i}_{+}^{\mathbf{n}}$ and $b<\mathbf{i}_{+}^{\mathbf{m}}$, where $\mathbf{i}_{+}^{\mathbf{n}}=\left(i^{n}\right)_{i \in \omega}$ and $\mathbf{i}_{+}^{\mathbf{m}}=\left(i^{m}\right)_{i \in \omega}$.

We define $O_{2} \mathbf{0}=(1 / 3) \mathbf{i}^{\mathbf{m}} \cdot O_{1} \mathbf{0}$ and $O_{3} \mathbf{0}=(1 / 3) \mathbf{i}^{\mathbf{n}} \cdot O_{1} \mathbf{0}$, where $\mathbf{i}^{\mathbf{n}}=\left(1 / i^{n}\right)_{i \in \omega}$ and $\mathbf{i}^{\mathbf{m}}=$ $\left(1 / i^{m}\right)_{i \in \omega}$. Then $\mathrm{Oa}=\mathrm{O}_{2} \mathbf{0}+a$ and $\mathrm{Ob}=\mathrm{O}_{3} \mathbf{0}+b$ are E-conical neighborhoods of $a$ and $b$, correspondingly. For these neighborhoods, we have

$$
\begin{aligned}
(O a) \cdot(O b) & =\left(O_{2} \mathbf{0}+a\right) \cdot\left(O_{3} \mathbf{0}+b\right) \subseteq O_{2} \mathbf{0} \cdot O_{3} \mathbf{0}+a \cdot O_{3} \mathbf{0}+b \cdot O_{2} \mathbf{0}+a \cdot b \\
& \subseteq\left(\frac{1}{9}\right) \cdot O_{1} \mathbf{0} \cdot O_{1} \mathbf{0}+\left(\frac{1}{3}\right)\left(a \cdot \mathbf{i}^{\mathbf{n}}\right) \cdot O_{1} \mathbf{0}+\left(\frac{1}{3}\right)\left(b \cdot \mathbf{i}^{\mathbf{m}}\right) \cdot O_{1} \mathbf{0}+a \cdot b \\
& \subseteq\left(\frac{1}{9}\right) \cdot O_{1} \mathbf{0}+\left(\frac{1}{3}\right) \cdot O_{1} \mathbf{0}+\left(\frac{1}{3}\right) \cdot O_{1} \mathbf{0}+a \cdot b \\
& \subseteq O_{1} \mathbf{0}+a \cdot b \subseteq O \mathbf{0}+a \cdot b=O(a \cdot b) .
\end{aligned}
$$

Thus, multiplication $a \cdot b$ in $\mathbf{E}^{\mathbf{D}} \mathbb{R}_{\omega}$ is a continuous mapping and $\mathbf{E}^{\mathbf{D}} \mathbb{R}_{\omega}$ with the topology $\delta_{c \mathrm{E}}$ is a topological linear algebra.

Theorem 3.42 is proved. 


\section{Conclusion}

Thus, a topological linear algebra of extended hypernumbers has been constructed with a Hausdorff topology in it. Topological properties made it possible to give (Theorem 3.25) an axiomatic description of extended hypernumbers using an extremality principle.

One can think of the further development of the theory of extrafunctions in several directions based on extended hypernumbers. First, a similar technique allows one to construct linear algebras for different types of extended extrafunctions (pointwise, compactwise, and extended distributions) with Hausdorff topology and similar to ordinary extrafunctions, and to develop a differential calculus in this nonlinear context.

Second, it looks beneficial to apply this theory to the problems of nonlinear differential equations. Specific properties of the theory of extended extrafunctions make it possible to achieve much more in this direction, allowing one to solve equations that have no solution even in distributions, than by means of the theory of generalized functions. Even in a linear case, the scope of soluble equations is essentially enlarged (Burgin and Ralston [11]). In a nonlinear situation, opportunities of extrafunction solutions grow much more.

Third, it would be advantageous to use the theory of extrafunctions for the development of the theory of operators and operator algebras. Extended hypernumbers allow one to define hypernorms not only in topological spaces but also in topological algebras. With respect to operators, this makes it possible to consider unbounded operators to a full extent and to study algebras of such operators.

\section{References}

[1] L. Berg, Construction of distribution algebras, Math. Nachr. 82 (1978), 255-262.

[2] G. Birkhoff and J. D. Lipson, Heterogeneous algebras, J. Combinatorial Theory 8 (1970), 115133.

[3] G. Boole, Treatise on the Calculus of Finite Differences, 2nd ed., Dover Publications, New York, 2003.

[4] M. Burgin, Extrafunctions, Distributions, and Nonsmooth Analysis, MRS Report 01-02, University of California, California, Mathematics Report Series, 2001, 47 pages.

[5] - Topological algebras with continuous operations, Dokl. Akad. Nauk SSSR 213 (1973), no. 3, 505-508, (translated from Russian).

[6] - Algebraic structures of distributions, Doklady of the Academy of Sciences of Ukraine (1987), no. 7, 5-9 (Russian and Ukrainian).

[7] _ Theory of hypernumbers and extrafunctions: functional spaces and differentiation, Discrete Dyn. Nat. Soc. 7 (2002), no. 3, 201-212.

[8] - Hyperfunctionals and generalized distributions, Stochastic Processes and Functional Analysis (A. C. Krinik and R. J. Swift, eds.), Lecture Notes in Pure and Appl. Math., vol. 238, Dekker, New York, 2004, pp. 81-119.

[9] — Theory of Real Hypernumbers: Properties and Axiomatization, Elsevier, preprint 0107062, 2001, 42 pages (electronic edition: http://www.sciencedirect.com/ preprintarchive).

[10] Unified Foundations of Mathematics, preprint Mathematics LO/0403186, 2004, 39 pages (electronic edition: http://arXiv.org).

[11] M. Burgin and J. Ralston, PDE and extrafunctions, Rocky Mountain J. Math. 34 (2004), no. 3, 849-867. 
[12] P. M. Cohn, Universal Algebra, Harper \& Row Publishers, New York, 1965.

[13] J.-F. Colombeau, A Mathematical Analysis Adapted to the Multiplication of Distributions, Springer, New York, 1986.

[14] A. Delcroix and D. Scarpalezos, Asymptotic scales, asymptotic algebras, Integral Transforms and Special Functions, vol. 6, no. 1-4, 1998, pp. 154-161.

[15] Yu. V. Egorov, A contribution to the theory of generalized functions, Russian Math. Surveys 45 (1990), no. 5, 1-49.

[16] B. Fisher, Products of generalized functions, Studia Math. 33 (1969), 227-230.

[17] V. Gontar, New theoretical approach of physico-chemical reactions dynamics with chaotic behavior, Chaos in Chemistry and Biochemistry (R. J. Field and L. Gyorgyi, eds.), World Scientific, London, 1993, pp. 225-247.

[18] P. R. Halmos, Algebraic Logic, Oxford University Press, New York, 1962.

[19] P. J. Higgins, Algebras with a scheme of operators, Math. Nachr. 27 (1963), no. 1-2, 115-132.

[20] N Notes on Categories and Groupoids, North-Holland: Van Nostrand Reinhold, 1973.

[21] C. Jordan, Calculus of Finite Differences, 3rd ed., Chelsea Publishing, New York, 1965.

[22] V. Kac and P. Cheung, Quantum Calculus, Springer, Berlin, 2002.

[23] L. H. Kauffman and R. A. Baadhio, eds., Quantum Topology, World Scientific Publishing, New Jersey, 1993.

[24] J. L. Kelley, General Topology, D. Van Nostrand, New York, 1955.

[25] K. Kuratowski, Topology. Vol. I, New edition, revised and augmented. Translated from the French by J. Jaworowski, Academic Press, New York, 1966.

[26] _ Topology. Vol. II, New edition, revised and augmented. Translated from the French by A. Kirkor, Academic Press, New York, 1968.

[27] A. G. Kurosh, General Algebra, Nauka Press, Moscow, 1974.

[28] L. LeBlanc, Nonhomogeneous polyadic algebras, Proc. Amer. Math. Soc. 13 (1962), no. 1, 59-65.

[29] B.-H. Li, Nonstandard analysis and multiplication of distributions in any dimension, Sci. Sinica Ser. A 28 (1978), no. 7, 716-726.

[30] A. Mallios, Topological Algebras. Selected Topics, North-Holland Mathematics Studies, vol. 124, North-Holland Publishing, Amsterdam, 1986.

[31] G. Matthiessen, A heterogeneous algebraic approach to some problems in automata theory, manyvalued logics and other topics, Contributions to General Algebra (Proc. Klagenfurt Conf., Klagenfurt, 1978), Heyn, Klagenfurt, 1979, pp. 193-211.

[32] G. McCarty, Topology: An Introduction with Application to Topological Groups, 2nd ed., Dover Publications, New York, 1988.

[33] Y. Meyer, Multiplication of distributions, Mathematical Analysis and Applications, Part B (L. Nachbin, ed.), Adv. in Math. Suppl. Stud., vol. 7, Academic Press, New York, 1981, pp. 603615.

[34] L. M. Milne-Thomson, The Calculus of Finite Differences, Macmillan, London, 1951.

[35] C. Nash, Topology and Physics—a Historical Essay, LANL, Preprint hep-th/9709135, 1997 (electronic edition: http://arXiv.org).

[36] G. Nicolis and I. Prigogine, Self-Organization in Non-Equilibrium Systems, Wiley-Interscience, New York, 1977.

[37] M. Oberguggenberger, Multiplication of Distributions and Applications to Partial Differential Equations, Pitman Research Notes in Mathematics Series, vol. 259, Longman Scientific \& Technical, Harlow, 1992.

[38] B. I. Plotkin, Universal Algebra, Algebraic Logic, and Databases, Nauka Press, Moscow, 1991.

[39] C. H. Richardson, An Introduction to the Calculus of Finite Differences, Van Nostrand, New York, 1954.

[40] E. E. Rosinger, Distributions and Non-Linear Partial Differential Equations, Lecture Notes in Mathematics, vol. 684, Springer, Berlin, 1978. 
[41] Non-Linear Partial Differential Equations. An Algebraic View of Generalized Solutions, North-Holland Mathematics Studies, vol. 164, North-Holland Publishing, Amsterdam, 1990.

[42] K. A. Ross, Elementary Analysis: The Theory of Calculus, Springer, New York, 1996.

[43] M. Spiegel, Calculus of Finite Differences and Differential Equations, McGraw-Hill, New York, 1971.

[44] E. Witten, Geometry and physics, Proceedings Internat. Congr. Math., 1986, vol. 1, American Mathematical Society, 1987, pp. 267-303.

[45] Geometry and quantum field theory, Proceedings AMS Centennial Symposium, 1988, pp. 479-491.

M. S. Burgin: Department of Mathematics, University of California, Los Angeles, 405 Hilgard Avenue, Los Angeles, CA 90095, USA

E-mail address: mburgin@math.ucla.edu 


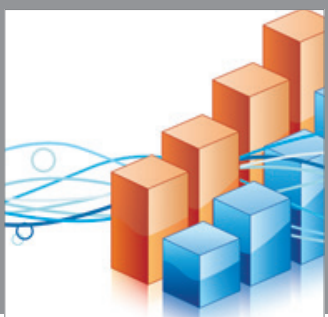

Advances in

Operations Research

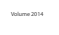

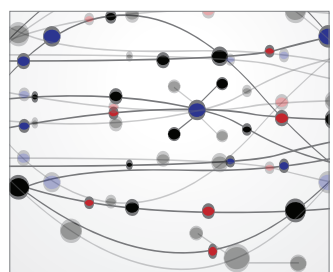

\section{The Scientific} World Journal
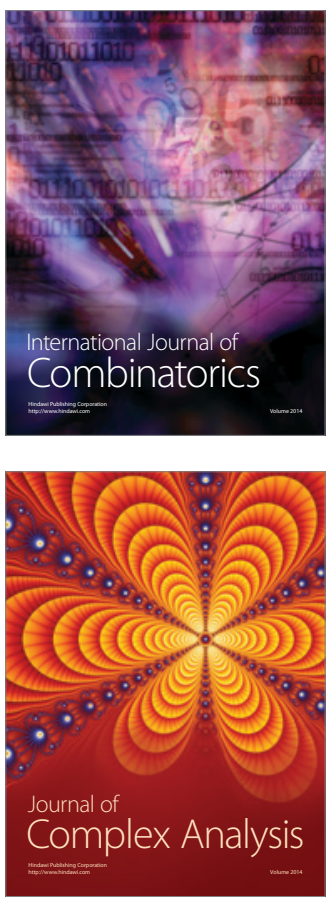

International Journal of

Mathematics and

Mathematical

Sciences
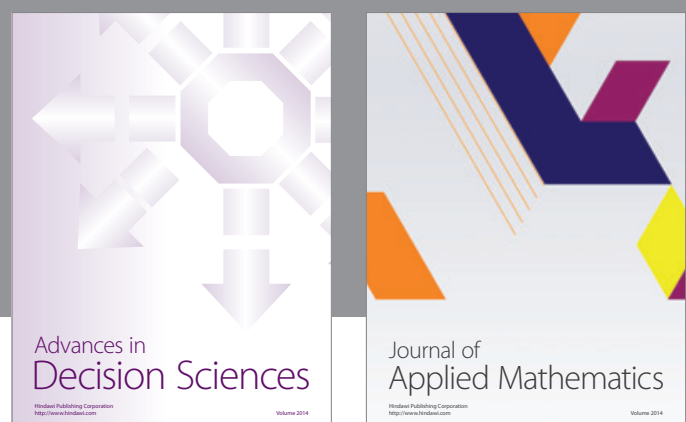

Journal of

Applied Mathematics
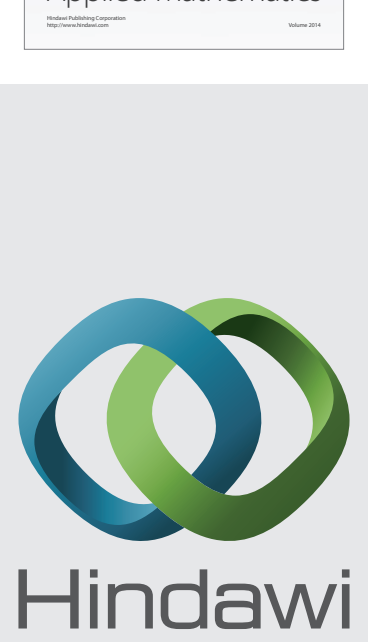

Submit your manuscripts at http://www.hindawi.com
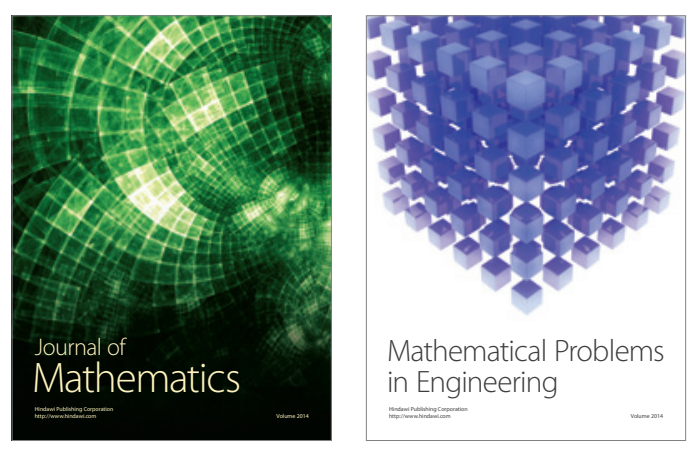

Mathematical Problems in Engineering
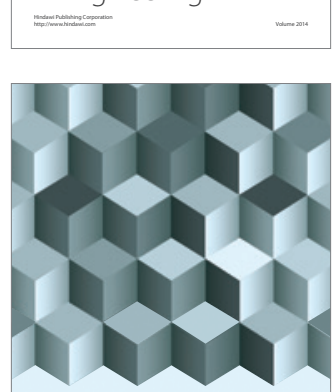

Journal of

Function Spaces
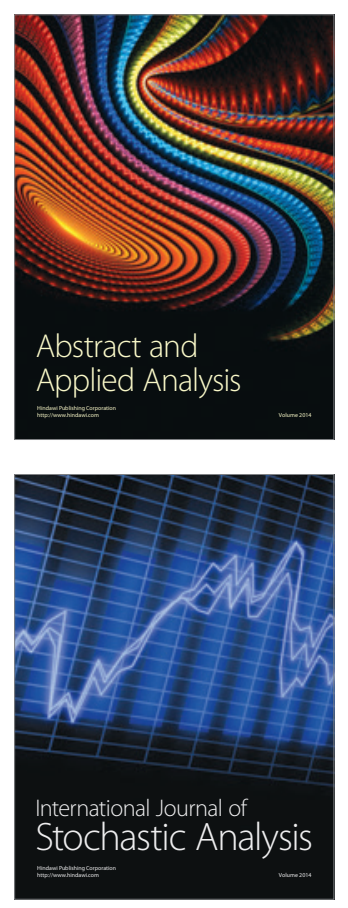

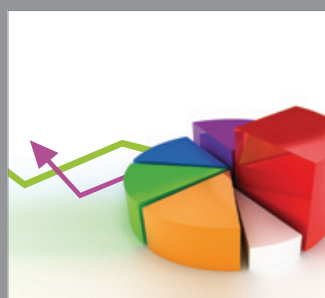

ournal of

Probability and Statistics

Promensencen
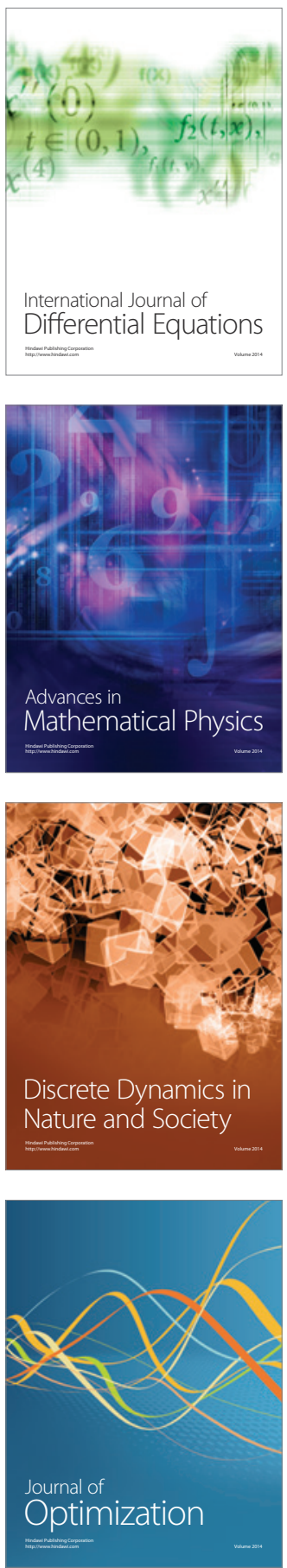